\title{
Beyond-Birthday-Bound Security for Tweakable Even-Mansour Ciphers with Linear Tweak and Key Mixing*
}

\author{
Benoît Cogliati ${ }^{\star \star}$ and Yannick Seurin ${ }^{\star \star \star}$
}

September 7, 2015

\begin{abstract}
The iterated Even-Mansour construction defines a block cipher from a tuple of public $n$-bit permutations $\left(P_{1}, \ldots, P_{r}\right)$ by alternatively xoring some $n$-bit round key $k_{i}, i=0, \ldots, r$, and applying permutation $P_{i}$ to the state. The tweakable Even-Mansour construction generalizes the conventional Even-Mansour construction by replacing the $n$-bit round keys by $n$-bit strings derived from a master key and a tweak, thereby defining a tweakable block cipher. Constructions of this type have been previously analyzed, but they were either secure only up to the birthday bound, or they used a nonlinear mixing function of the key and the tweak (typically, multiplication of the key and the tweak seen as elements of some finite field) which might be costly to implement. In this paper, we tackle the question of whether it is possible to achieve beyond-birthday-bound security for such a construction by using only linear operations for mixing the key and the tweak into the state. We answer positively, describing a 4-round construction with a $2 n$-bit master key and an $n$-bit tweak which is provably secure in the Random Permutation Model up to roughly $2^{2 n / 3}$ adversarial queries.
\end{abstract}

Keywords: tweakable block cipher, iterated Even-Mansour cipher, key-alternating cipher, beyond-birthday-bound security

\section{Introduction}

BACKGRound. A block cipher with key space $\mathcal{K}$ and message space $\mathcal{M}$ is a family of permutations of $\mathcal{M}$ indexed by the key $\mathbf{k} \in \mathcal{K}$. A tweakable block cipher (TBC) takes an additional (potentially public) input parameter $\mathbf{t} \in \mathcal{T}$ called a tweak aiming at providing inherent variability in about the same way an IV or nonce brings variability to an encryption scheme. Some block ciphers such as the Hasty Pudding Cipher [Sch98], Mercy [Cro00], or Threefish (the block cipher underlying the Skein hash function $\left[\mathrm{FLS}^{+} 10\right]$ ) were designed so as to natively support tweaks. The syntax and security requirements for tweakable block ciphers were formally articulated in a seminal paper by Liskov, Rivest and Wagner [LRW02]. Since then, TBCs have found multiple applications such as (tweakable) length-preserving encryption modes [HR03, HR04], online ciphers [RZ11, ABL $\left.{ }^{+} 13\right]$, and authenticated encryption modes [LRW02, RBB03, Rog04].

Liskov et al. [LRW02] also proposed two generic constructions of a TBC from a standard block cipher, achieving security up to the so-called birthday bound, i.e., when the adversary is allowed at most roughly $2^{n / 2}$ queries to the encryption or decryption oracle, where $n$ is the block size (that is, the message space of the TBC is $\mathcal{M}=\{0,1\}^{n}$ ). The "black-box" design strategy (i.e., building a TBC on top of an existing standard block cipher, in a black-box

\footnotetext{
* (C) IACR 2015. This is the full version of the article submitted by the authors to the IACR and to SpringerVerlag in September 2015, which appears in the proceedings of ASIACRYPT 2015.

${ }^{\star \star}$ University of Versailles, France. E-mail: benoitcogliati@hotmail.fr

$\star \star \star$ ANSSI, Paris, France. E-mail: yannick.seurin@m4x.org
} 
way) has since then been the main avenue of research. Earlier proposals, such as XEX [Rog04] and variants [Min06, CS06] were related to the second of the two original proposals of Liskov et al., and were limited to birthday-bound security as well. Recently, a number of constructions achieving beyond-birthday-bound security have emerged, such as Minematsu's construction [Min09], the CLRW construction [LST12, LS14, Pro14], and two constructions by Mennink [Men15]. All those constructions enjoy a security proof in the standard model (i.e., assuming that the underlying block cipher is a pseudorandom permutation), except for Mennink's constructions that were analyzed in the ideal cipher model.

Tweaking Even-Mansour Ciphers. Unfortunately, none of the currently known black-box TBC constructions with beyond-birthday-bound security can be deemed truly practical (even though some of them might come close to it [Men15]). Hence, it might be beneficial to "open the hood" and to study how to build a TBC from some lower level primitive than a full-fledged conventional block cipher, e.g., a pseudorandom function or a public permutation. For example, Goldenberg et al. [GHL $\left.{ }^{+} 07\right]$ investigated how to include a tweak in Feistel ciphers. This was extended to generalized Feistel ciphers by Mitsuda and Iwata [MI08]. Recently, a similar study was undertaken for the second large class of block ciphers besides Feistel ciphers, namely key-alternating ciphers [DR01], a super-class of Substitution-Permutation Networks (SPNs). An $r$-round key-alternating cipher based on a tuple of public $n$-bit permutations $\left(P_{1}, \ldots, P_{r}\right)$ maps a plaintext $x \in\{0,1\}^{n}$ to the ciphertext defined as

$$
y=k_{r} \oplus P_{r}\left(k_{r-1} \oplus P_{r-1}\left(\cdots P_{2}\left(k_{1} \oplus P_{1}\left(k_{0} \oplus x\right)\right) \cdots\right)\right)
$$

where the $n$-bit round keys $k_{0}, \ldots, k_{r}$ are either independent or derived from a master key $\mathbf{k}$. When the $P_{i}$ 's are modeled as public permutation oracles, construction (1) is also referred to as the (iterated) Even-Mansour construction, in reference to Even and Mansour who pioneered the analysis of this construction in the Random Permutation Model [EM97]. While Even and Mansour limited themselves to proving birthday-bound security in the case $r=1$, larger numbers of rounds were studied in subsequent works [BKL ${ }^{+} 12$, Ste12, LPS12]. The general case has been recently (tightly) settled by Chen and Steinberger [CS14], who proved that the $r$-round iterated Even-Mansour cipher with $r$-wise independent round keys ensures security up to roughly $2^{\frac{r n}{r+1}}$ adversarial queries.

In order to incorporate a tweak $\mathbf{t}$ in the iterated Even-Mansour construction, it is tantalizing to generalize (1) by replacing round keys $k_{i}$ by some function $f_{i}(\mathbf{k}, \mathbf{t})$ of the master key $\mathbf{k}$ and the tweak $\mathbf{t}$ (see Figure 1). We will refer to such a construction as a Tweakable Even-Mansour (TEM) construction. ${ }^{1}$ This is exactly the spirit of the TWEAKEY framework introduced by Jean et al. [JNP14]. In fact, these authors go one step further and propose to unify the key and tweak inputs into what they dub the tweakey. The main topic of this paper being provable security (in the traditional model where the key is secret and the tweak is chosen by the adversary), we will not make such a bold move here, since we are not aware of any formal security model adequately capturing what Jean et al. had in mind.

\footnotetext{
${ }^{1}$ We warn that the naming Tweakable Even-Mansour construction was previously used by the designers of Minalpher $\left[\mathrm{STA}^{+}{ }^{14}\right]$, a candidate to the CAESAR competition, to designate a permutation-based variant of Rogaway's XEX construction [Rog04], i.e., a 1-round Even-Mansour construction where the derivation functions $f_{0}$ and $f_{1}$ applied to $(\mathbf{k}, \mathbf{t})$ are allowed to depend on the internal permutation $P_{1}$ (something we do not consider in this paper).
} 
The investigation of the theoretical soundness of this design strategy was initiated in three recent papers. First, Cogliati and Seurin [CS15], and independently Farshim and Procter [FP15], analyzed the simple case of an $n$-bit key $k$ and an $n$-bit tweak $t$ simply xored together at each round, i.e., $f_{i}(k, t)=k \oplus t$ for each $i=0, \ldots, r{ }^{2}$ They gave attacks up to two rounds, and proved birthday-bound security for three rounds. In fact, the security of this construction caps at $2^{n / 2}$ queries independently of the number of rounds. Indeed, it can be written $\widetilde{E}(k, t, x)=E(k \oplus t, x)$, where $E$ is the conventional iterated Even-Mansour cipher with the trivial key-schedule (i.e., the same round key is xored between each round), and by a result of Bellare and Kohno [BK03, Corollary 5.7], a tweakable block cipher of this form can never offer more than $\kappa / 2$ bits of security, where $\kappa$ is the key-length of $E$ (i.e., $\kappa=n$ in the case at hand). Hence, if we want beyond-birthday-bound security, we have no choice but to consider more complex functions $f_{i}$ (at the bare minimum, these functions, even if linear, should prevent the TBC construction from being of the form $E(k \oplus t, x)$ for some block cipher $E$ with $n$-bit keys).

This was undertaken by Cogliati, Lampe, and Seurin [CLS15], who considered nonlinear ways of mixing the key and the tweak. More specifically, they studied the case where $f_{i}(\mathbf{k}, t)=$ $H_{k_{i}}(t)$, where the family of functions $\left(H_{k}\right)$ is uniform and almost XOR-universal, and the master key is $\mathbf{k}=\left(k_{0}, \ldots, k_{r}\right)$. A classical example is multiplication-based hashing, i.e., $f_{i}(\mathbf{k}, t)=k_{i} \otimes t$, where $\otimes$ denotes the multiplication in the finite field $\mathbb{F}_{2^{n}}$, the tweak $t=0$ being forbidden. Cogliati et al. showed that one round is secure up to the birthday bound, and that two rounds are secure up to roughly $2^{2 n / 3}$ adversarial queries. ${ }^{3}$ They also provided a (non-tight) asymptotic security bound improving as the number of rounds grows. However, implementing a xor-universal hash function might be costly, and linear functions $f_{i}$ 's would be highly preferable for obvious efficiency reasons.

OuR Results. In this paper, we ask whether it is possible to come with a tweakable EvenMansour construction achieving both:

1. a linear mixing of the tweak and the key to the state;

2. beyond-birthday-bound security.

We answer positively, by providing a construction with $2 n$-bit keys and $n$-bit tweaks. The starting point is the 4-round iterated Even-Mansour construction with a $2 n$-bit master key $\left(k_{0}, k_{1}\right), k_{0}$ and $k_{1}$ being both $n$ bits, and what we call the "alternating" key schedule, namely round keys are $k_{0}, k_{1}, k_{0}$, etc. This is for example how LED-128 is designed [GPPR11]. To turn this block cipher into a tweakable Even-Mansour construction, we simply add the $n$-bit tweak $t$ between each permutation (see Figure 2). In other words, if we denote $E\left(\left(k_{0}, k_{1}\right), x\right)$ the conventional Even-Mansour cipher with alternating round keys, the tweakable construction that we consider can be written

$$
\widetilde{E}\left(\left(k_{0}, k_{1}\right), t, x\right)=E\left(\left(k_{0} \oplus t, k_{1} \oplus t\right), x\right) .
$$

We prove that this construction is secure up to roughly $2^{2 n / 3}$ adversarial queries. Unsurprisingly, and as in many previous works, our proof uses Patarin's H-coefficients technique [Pat08, CS14].

\footnotetext{
${ }^{2}$ Actually, the results of [CS15, FP15] were stated in terms of xor-induced related-key security of the (conventional) iterated Even-Mansour cipher, but in this case this is equivalent to standard (i.e., single-key) security of the corresponding tweakable construction.

${ }^{3}$ More precisely, the birthday-bound result applies to the variant of the construction were the same key is used before and after permutation $P_{1}$, and the $2^{2 n / 3}$-security bound applies to the cascade of this construction with two independent keys and two independent permutations.
} 
In particular, we rely on a key lemma by Cogliati et al. [CLS15] to analyze so-called good transcripts.

Application to Related-Key Security. Our result can be rephrased in terms of relatedkey security [BK03] of the conventional Even-Mansour cipher: the 4-round conventional EvenMansour cipher with the alternating key-schedule is secure up to roughly $2^{2 n / 3}$ adversarial queries against related-key attacks for the set of related-key deriving functions

$$
\Phi^{2-\oplus \stackrel{\text { def }}{=}}\left\{\left(k_{0}, k_{1}\right) \mapsto\left(k_{0} \oplus \Delta, k_{1} \oplus \Delta\right): \Delta \in\{0,1\}^{n}\right\} .
$$

Note that this set is more restrictive than the set $\Phi^{\oplus}$ that would allow to xor an arbitrary $2 n$-bit string to the master key $\left(k_{0}, k_{1}\right)$. It remains an open problem (already stated in [CS15]) to find an Even-Mansour construction provably secure beyond the birthday bound against $\Phi^{\oplus}$-related-key attacks.

Open Problems. We propose three challenging open problems, the first two being restricted to the case of $n$-bit tweaks. First, what would be the analogue of the Chen-Steinberger result [CS14] in the tweakable setting? In more details, we know how to deliver $n / 2$ bits of security with an $n$-bit master key [CS15, FP15] and this paper shows how to reach $2 n / 3$ bits of security with a $2 n$-bit master key. Hence, it is natural to ask whether one can obtain $r n /(r+1)$ bits of security from an $r n$-bit master key for $r>2$, and what would be the adequate number of rounds and the corresponding (linear) "tweak-and-key" schedule. Second, Chen et al. $\left[\mathrm{CLL}^{+} 14\right]$ showed that the 2-round conventional Even-Mansour construction can provably deliver $2 n / 3$ bits of security even with an $n$-bit master key (for example, when the two inner permutations are independent, the trivial key-schedule is sufficient). Again, what would be the analogue of this result in the tweakable setting? Can we design a TEM construction with an $n$-bit master key and an $n$-bit tweak delivering $2 n / 3$ bits of security, or even more? Finally, it is natural to ask whether one can extend the construction of this paper to handle larger tweaks, in particular $2 n$-bit tweaks. We show in Appendix B that the naive way of proceeding, namely adding alternatively $t_{0}$ and $t_{1}$, is insecure for four rounds. Hence, this seems to require at least five rounds.

We also remark that attacks against the (conventional) iterated Even-Mansour cipher with the alternating key-schedule have been investigated by Dinur et al. [DDKS14]. It would be interesting to study whether these attacks can be adapted (and potentially improved) in the tweakable setting.

Organization. In Section 2, we introduce the notation, the security definitions, and give some background on the H-coefficients technique. Our main result is proved in Section 3.

\section{Preliminaries}

\subsection{Notation and General Definitions}

General Notation. In all the following, we fix an integer $n \geq 1$ and denote $N=2^{n}$. For integers $1 \leq b \leq a$, we will write $(a)_{b}=a(a-1) \cdots(a-b+1)$ and $(a)_{0}=1$ by convention. The set of all permutations of $\{0,1\}^{n}$ will be denoted $\mathrm{P}(n)$. 


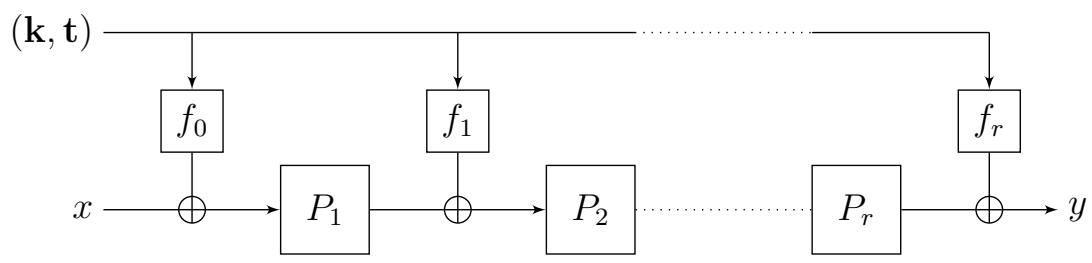

Fig. 1. The $r$-round tweakable Even-Mansour construction based on a tuple of public permutations $\left(P_{1}, \ldots, P_{r}\right)$.

Tweakable Block Ciphers. A tweakable block cipher with key space $\mathcal{K}$, tweak space $\mathcal{T}$, and message space $\mathcal{M}$ is a mapping $\widetilde{E}: \mathcal{K} \times \mathcal{T} \times \mathcal{M} \rightarrow \mathcal{M}$ such that for any key $k \in \mathcal{K}$ and any tweak $t \in \mathcal{T}, x \mapsto \widetilde{E}(k, t, x)$ is a permutation of $\mathcal{M}$. We denote $\operatorname{TBC}(\mathcal{K}, \mathcal{T}, n)$ the set of all tweakable block ciphers with key space $\mathcal{K}$, tweak space $\mathcal{T}$, and message space $\{0,1\}^{n}$. A tweakable permutation with tweak space $\mathcal{T}$ and message space $\mathcal{M}$ is a mapping $\widetilde{P}: \mathcal{T} \times \mathcal{M} \rightarrow \mathcal{M}$ such that for any tweak $t \in \mathcal{T}, x \mapsto \widetilde{P}(t, x)$ is a permutation of $\mathcal{M}$. We denote $\operatorname{TP}(\mathcal{T}, n)$ the set of all tweakable permutations with tweak space $\mathcal{T}$ and message space $\{0,1\}^{n}$.

Tweakable Even-Mansour Constructions. Fix integers $n, r \geq 1$. Let $\mathcal{K}$ and $\mathcal{T}$ be two sets, and let $\mathbf{f}=\left(f_{0}, \ldots, f_{r}\right)$ be a $(r+1)$-tuple of functions from $\mathcal{K} \times \mathcal{T}$ to $\{0,1\}^{n}$. The $r$-round tweakable Even-Mansour construction TEM $[n, r, \mathbf{f}]$ specifies, from an $r$-tuple $\mathbf{P}=\left(P_{1}, \ldots, P_{r}\right)$ of permutations of $\{0,1\}^{n}$, a tweakable block cipher with key space $\mathcal{K}$, tweak space $\mathcal{T}$, and message space $\{0,1\}^{n}$, simply denoted TEM ${ }^{\mathbf{P}}$ in the following (parameters $[n, r, \mathbf{f}]$ will always be clear from the context) which maps a key $\mathbf{k} \in \mathcal{K}$, a tweak $\mathbf{t} \in \mathcal{T}$, and a plaintext $x \in\{0,1\}^{n}$ to the ciphertext defined as (see Figure 1):

$$
\operatorname{TEM}^{\mathbf{P}}(\mathbf{k}, \mathbf{t}, x)=f_{r}(\mathbf{k}, \mathbf{t}) \oplus P_{r}\left(f_{r-1}(\mathbf{k}, \mathbf{t}) \oplus P_{r-1}\left(\cdots P_{1}\left(f_{0}(\mathbf{k}, \mathbf{t}) \oplus x\right) \cdots\right)\right) .
$$

We will denote $\operatorname{TEM}_{\mathbf{k}}^{\mathbf{P}}$ the mapping taking as input $(\mathbf{t}, x) \in \mathcal{T} \times\{0,1\}^{n}$ and returning $\operatorname{TEM}^{\mathbf{P}}(\mathbf{k}, \mathbf{t}, x)$.

We will mostly be interested in the case where $\mathcal{K}=\left(\{0,1\}^{n}\right)^{a}$ and $\mathcal{T}=\left(\{0,1\}^{n}\right)^{b}$ for integers $a, b \geq 1$. In this setting, we will denote $\mathbf{k}=\left(k_{0}, \ldots, k_{a-1}\right)$ and $\mathbf{t}=\left(t_{0}, \ldots, t_{b-1}\right)$, all $k_{i}$ 's and $t_{j}$ 's being $n$-bit strings, or simply $\mathbf{k}=k$, resp. $\mathbf{t}=t$ when $a=1$, resp. $b=1$. When all $f_{i}$ 's are linear over $\left(\{0,1\}^{n}\right)^{a+b}$, we say that the construction has linear tweak and key mixing.

Previously Studied Constructions. Two types of TEM constructions have already been studied. In [CS15], Cogliati and Seurin considered the simplest case where $a=b=1$ ( $n$-bit keys and $n$-bit tweaks) and $f_{i}(k, t)=k \oplus t$ for each $i=0, \ldots, r$. This construction has linear tweak and key mixing, and is secure up to $2^{n / 2}$ adversarial queries starting from $r=3$. (The results of [CS15] were formulated in terms of xor-induced related-key attacks against the conventional iterated Even-Mansour construction, but in this simple case the two security notions are in fact equivalent.) In [CLS15], Cogliati, Lampe, and Seurin studied a large class of nonlinear mixing functions, in particular, for $n$-bit tweaks, finite field multiplication-based ones, i.e., $f(k, t)=k \otimes t$, or more generally, for $b n$-bit tweaks, polynomial hashing-based functions, i.e., $f\left(k,\left(t_{0}, \ldots, t_{b-1}\right)\right)=\sum_{i=0}^{b-1} k^{i+1} \otimes t_{i}$. 


\subsection{Security Definitions}

Fix some family of functions $\mathbf{f}=\left(f_{0}, \ldots, f_{r}\right)$ from $\mathcal{K} \times \mathcal{T}$ to $\{0,1\}^{n}$. To study the security of the construction TEM $[n, r, \mathbf{f}]$ in the Random Permutation Model, we consider a distinguisher $\mathcal{D}$ which interacts with $r+1$ oracles that we denote generically $\left(\widetilde{P}_{0}, P_{1}, \ldots, P_{r}\right)$, where syntactically $\widetilde{P}_{0}$ is a tweakable permutation with tweak space $\mathcal{T}$ and message space $\{0,1\}^{n}$, and $P_{1}, \ldots, P_{r}$ are permutations of $\{0,1\}^{n}$. The goal of $\mathcal{D}$ is to distinguish two "worlds": the so-called real world, where $\mathcal{D}$ interacts with $\left(\operatorname{TEM}_{\mathbf{k}}^{\mathbf{P}}, \mathbf{P}\right)$, where $\mathbf{P}=\left(P_{1}, \ldots, P_{r}\right)$ is a tuple of public random permutations and the key $\mathbf{k}$ is drawn uniformly at random from $\mathcal{K}$, and the so-called ideal world $\left(\widetilde{P}_{0}, \mathbf{P}\right)$, where $\widetilde{P}_{0}$ is a uniformly random tweakable permutation and $\mathbf{P}$ is a tuple of random permutations of $\{0,1\}^{n}$ independent from $\widetilde{P}_{0}$. We will refer to $\widetilde{P}_{0}$ as the construction oracle and to $P_{1}, \ldots, P_{r}$ as the inner permutation oracles.

The distinguishing advantage of a distinguisher $\mathcal{D}$ is defined as

$$
\operatorname{Adv}(\mathcal{D}) \stackrel{\text { def }}{=}\left|\operatorname{Pr}\left[\mathcal{D}^{\mathrm{TEM}} \mathbf{k}_{\mathbf{k}}^{\mathbf{P}}, \mathbf{P}=1\right]-\operatorname{Pr}\left[\mathcal{D}^{\widetilde{P}_{0}, \mathbf{P}}=1\right]\right|,
$$

where the first probability is taken over the random choice of $\mathbf{k}$ and $\mathbf{P}$, and the second probability is taken over the random choice of $\widetilde{P}_{0}$ and $\mathbf{P}$. In all the following, we consider computationally unbounded distinguishers, and hence we can assume wlog that they are deterministic. We also assume that they never make pointless queries (i.e., queries whose answers can be unambiguously deduced from previous answers). The distinguisher is allowed to query all oracles adaptively in both directions; this corresponds to adaptive chosen-plaintext and ciphertext attacks (CCA).

For non-negative integers $q_{c}$ and $q_{p}$, we define the insecurity of the $\operatorname{TEM}[n, r, \mathbf{f}]$ construction against CCA-attacks as

$$
\mathbf{A d v}_{\mathrm{TEM}[n, r, \mathbf{f}]}^{\mathrm{cca}}\left(q_{c}, q_{p}\right)=\max _{\mathcal{D}} \mathbf{A d v}(\mathcal{D}),
$$

where the maximum is taken over all distinguishers making exactly $q_{c}$ queries to the construction oracle and exactly $q_{p}$ queries to each inner permutation oracle.

\subsection{The H-Coefficients Technique}

As in many previous works [CS14, $\mathrm{CLL}^{+} 14$, CS15, CLS15], our security proof will use the H-coefficients technique [Pat08], which we explain here.

TRANSCRIPT. Recall that the distinguisher $\mathcal{D}$ interacts with a tuple of $r+1$ oracles denoted $\left(\widetilde{P}_{0}, P_{1}, \ldots, P_{r}\right)$. In the real world, the construction oracle $\widetilde{P}_{0}$ is $\operatorname{TEM}_{\mathbf{k}}^{\mathbf{P}}$ where $\mathbf{P}=\left(P_{1}, \ldots, P_{r}\right)$ and $\mathbf{k}$ is random, whereas in the ideal world it is a random tweakable permutation independent from $\left(P_{1}, \ldots, P_{r}\right)$. From the interaction of $\mathcal{D}$ with these oracles, we define the queries transcript $\left(\mathcal{Q}_{C}, \mathcal{Q}_{P_{1}}, \ldots, \mathcal{Q}_{P_{r}}\right)$ of the attack as follows. The list $\mathcal{Q}_{C}$ records the queries to the construction oracle: if $\mathcal{D}$ made either a direct query $(\mathbf{t}, x)$ to the construction oracle $\widetilde{P}_{0}$ which was answered by $y$, or an inverse query $(\mathbf{t}, y)$ which was answered by $x$, then the triple $(\mathbf{t}, x, y) \in \mathcal{T} \times\{0,1\}^{n} \times$ $\{0,1\}^{n}$ is added to $\mathcal{Q}_{C}$. Similarly, for $1 \leq i \leq r, \mathcal{Q}_{P_{i}}$ contains all pairs $(u, v) \in\{0,1\}^{n} \times\{0,1\}^{n}$ such that $\mathcal{D}$ made either a direct query $u$ to permutation $P_{i}$ which was answered by $v$, or an inverse query $v$ which was answered by $u$. Note that queries are recorded in a directionless and unordered way, but by our assumption that the distinguisher is deterministic, the raw 
interaction of $\mathcal{D}$ with its oracles can unambiguously be reconstructed from the queries transcript (see e.g. [CS14] for more details). Note also that by our assumption that $\mathcal{D}$ never makes pointless queries, each query to the construction oracle results in a distinct triple in $\mathcal{Q}_{C}$, and each query to $P_{i}$ results in a distinct pair in $\mathcal{Q}_{P_{i}}$. Moreover, since we assume that the distinguisher always makes the maximal number of allowed queries to each oracle, one has $\left|\mathcal{Q}_{C}\right|=q_{c}$ and $\left|\mathcal{Q}_{P_{i}}\right|=q_{p}$ for $1 \leq i \leq r$. In all the following, we also denote $m$ the number of distinct tweaks appearing in $\mathcal{Q}_{C}$, and $q_{i}$ the number of queries for the $i$-th tweak, $1 \leq i \leq m$, ordering the tweaks arbitrarily. Note that one always has $\sum_{i=1}^{m} q_{i}=q_{c}$, even though $m$ may depend on the answers received from the oracles.

A queries transcript is said attainable (with respect to some fixed distinguisher $\mathcal{D}$ ) if there exists oracles $\left(\widetilde{P}_{0}, \mathbf{P}\right)$ such that the interaction of $\mathcal{D}$ with $\left(\widetilde{P}_{0}, \mathbf{P}\right)$ results in this transcript (in other words, the probability to obtain this transcript in the ideal world is non-zero). Moreover, in order to have a simple definition of bad transcripts, the actual key $\mathbf{k}$ is revealed to the adversary at the end of the experiment if we are in the real world, while in the ideal world, a "dummy" key $\mathbf{k} \leftarrow_{\$} \mathcal{K}$ is simply drawn uniformly at random independently from the answers of the oracle $\widetilde{P}_{0}$ (this is obviously without loss of generality since this can only help the distinguisher and increase its advantage). All in all, a transcript $\tau$ is a tuple $\tau=\left(\mathcal{Q}_{C}, \mathcal{Q}_{P_{1}}, \ldots, \mathcal{Q}_{P_{r}}, \mathbf{k}\right)$, and we say that a transcript is attainable if the corresponding queries transcript $\left(\mathcal{Q}_{C}, \mathcal{Q}_{P_{1}}, \ldots, \mathcal{Q}_{P_{r}}\right)$ is attainable. We denote $\Theta$ the set of attainable transcripts. In all the following, we denote $T_{\text {re }}$, resp. $T_{\text {id }}$, the probability distribution of the transcript $\tau$ induced by the real world, resp. the ideal world (note that these two probability distributions depend on the distinguisher). By extension, we use the same notation to denote a random variable distributed according to each distribution. The main lemma of the H-coefficients technique is the following one (see e.g. $\left[\mathrm{CS} 14, \mathrm{CLL}^{+} 14\right]$ for the proof).

Lemma 1. Fix a distinguisher $\mathcal{D}$. Let $\Theta=\Theta_{\text {good }} \sqcup \Theta_{\text {bad }}$ be a partition of the set of attainable transcripts. Assume that there exists $\varepsilon_{1}$ such that for any $\tau \in \Theta_{\text {good }}$, one has ${ }^{4}$

$$
\frac{\operatorname{Pr}\left[T_{\mathrm{re}}=\tau\right]}{\operatorname{Pr}\left[T_{\mathrm{id}}=\tau\right]} \geq 1-\varepsilon_{1}
$$

and that there exists $\varepsilon_{2}$ such that $\operatorname{Pr}\left[T_{\mathrm{id}} \in \Theta_{\mathrm{bad}}\right] \leq \varepsilon_{2}$. Then $\operatorname{Adv}(\mathcal{D}) \leq \varepsilon_{1}+\varepsilon_{2}$.

Useful Observations. We end this section with some useful preliminary observations. First, we introduce some additional notation. Given a permutation queries transcript $\mathcal{Q}$ and a permutation $P$, we say that $P$ extends $\mathcal{Q}$, denoted $P \vdash \mathcal{Q}$, if $P(u)=v$ for all $(u, v) \in \mathcal{Q}$. By extension, given a tuple of permutation queries transcripts $\mathcal{Q}_{\mathbf{P}}=\left(\mathcal{Q}_{P_{1}}, \ldots, \mathcal{Q}_{P_{r}}\right)$ and a tuple of permutations $\mathbf{P}=\left(P_{1}, \ldots, P_{r}\right)$, we say that $\mathbf{P}$ extends $\mathcal{Q}_{\mathbf{P}}$, denoted $\mathbf{P} \vdash \mathcal{Q}_{\mathbf{P}}$, if $P_{i} \vdash \mathcal{Q}_{P_{i}}$ for each $i=1, \ldots, r$. Note that for a permutation transcript of size $q_{p}$, one has

$$
\operatorname{Pr}\left[P \leftarrow{ }_{\$} \mathrm{P}(n): P \vdash \mathcal{Q}\right]=\frac{1}{(N)_{q_{p}}} .
$$

Similarly, given a tweakable permutation transcript $\widetilde{\mathcal{Q}}$ and a tweakable permutation $\widetilde{P}$, we say that $\widetilde{P}$ extends $\widetilde{\mathcal{Q}}$, denoted $\widetilde{P} \vdash \widetilde{\mathcal{Q}}$, if $\widetilde{P}(t, x)=y$ for all $(t, x, y) \in \widetilde{\mathcal{Q}}$. For a tweakable

\footnotetext{
${ }^{4}$ Recall that for an attainable transcript, one has $\operatorname{Pr}\left[T_{\text {id }}=\tau\right]>0$.
} 
permutation transcript $\widetilde{\mathcal{Q}}$ with $m$ distinct tweaks and $q_{i}$ queries corresponding to the $i$-th tweak, one has

$$
\operatorname{Pr}\left[\widetilde{P} \leftarrow_{\$} \operatorname{TP}(\mathcal{T}, n): \widetilde{P} \vdash \widetilde{\mathcal{Q}}\right]=\prod_{i=1}^{m} \frac{1}{(N)_{q_{i}}} .
$$

It is easy to see that the interaction of a distinguisher $\mathcal{D}$ with oracles $\left(\widetilde{P}_{0}, P_{1}, \ldots, P_{r}\right)$ yields any attainable queries transcript $\left(\mathcal{Q}_{C}, \mathcal{Q}_{\mathbf{P}}\right)$ with $\mathcal{Q}_{\mathbf{P}}=\left(\mathcal{Q}_{P_{1}}, \ldots, \mathcal{Q}_{P_{r}}\right)$ iff $\widetilde{P}_{0} \vdash \mathcal{Q}_{C}$ and $P_{i} \vdash \mathcal{Q}_{P_{i}}$ for $1 \leq i \leq r$. In the ideal world, the key $\mathbf{k}$, the permutations $P_{1}, \ldots, P_{r}$, and the tweakable permutation $\widetilde{P}_{0}$ are all uniformly random and independent, so that, by (2) and (3), the probability of getting any attainable transcript $\tau=\left(\mathcal{Q}_{C}, \mathcal{Q}_{\mathbf{P}}, \mathbf{k}\right)$ in the ideal world is

$$
\operatorname{Pr}\left[T_{\mathrm{id}}=\tau\right]=\frac{1}{|\mathcal{K}|} \times\left(\frac{1}{(N)_{q_{p}}}\right)^{r} \times \prod_{i=1}^{m} \frac{1}{(N)_{q_{i}}} .
$$

In the real world, the probability to obtain $\tau$ is

$$
\operatorname{Pr}\left[T_{\mathrm{re}}=\tau\right]=\frac{1}{|\mathcal{K}|} \times\left(\frac{1}{(N)_{q_{p}}}\right)^{r} \times \operatorname{Pr}\left[\mathbf{P} \leftarrow_{\$}(\mathrm{P}(n))^{r}: \operatorname{TEM}_{\mathbf{k}}^{\mathbf{P}} \vdash \mathcal{Q}_{C} \mid \mathbf{P} \vdash \mathcal{Q}_{\mathbf{P}}\right] .
$$

Let

$$
\mathrm{p}(\tau) \stackrel{\text { def }}{=} \operatorname{Pr}\left[\mathbf{P} \leftarrow_{\$}(\mathrm{P}(n))^{r}: \mathrm{TEM}_{\mathbf{k}}^{\mathbf{P}} \vdash \mathcal{Q}_{C} \mid \mathbf{P} \vdash \mathcal{Q}_{\mathbf{P}}\right]
$$

Then we have

$$
\frac{\operatorname{Pr}\left[T_{\mathrm{re}}=\tau\right]}{\operatorname{Pr}\left[T_{\mathrm{id}}=\tau\right]}=\mathrm{p}(\tau) / \prod_{i=1}^{m} \frac{1}{(N)_{q_{i}}} .
$$

Hence, applying Lemma 1 will require three steps: first, define good and bad transcripts, then upper bound the probability of bad transcripts in the ideal world, and finally lower bound the real world probability $\mathrm{p}(\tau)$ when $\tau$ is good in order to use Eq. (4).

\subsection{An Extended Sum-Capture Lemma}

To upper bound the probability of getting a bad transcript in the ideal world, we will need a generalization of the sum-capture theorem from $\left[\mathrm{CLL}^{+} 14\right]$ (that applied to a random permutation) to the case of a family of random permutations (in other words, a random tweakable permutation).

We denote $\mathrm{GL}(n)$ the general linear group of degree $n$ over $\mathbb{F}_{2}$, i.e., the set of all automorphisms (linear bijective mappings) of $\mathbb{F}_{2}^{n}$.

Lemma 2. Fix an automorphism $\Gamma \in \mathrm{GL}(n)$ and a non-empty set $\mathcal{T}$. Let $\widetilde{P}$ be a uniformly random tweakable permutation in $\operatorname{TP}(\mathcal{T}, n)$, and let $\mathcal{A}$ be some probabilistic algorithm making exactly $q$ (two-sided) adaptive queries to $\widetilde{P}$. Let $\widetilde{\mathcal{Q}}=\left(\left(t_{1}, x_{1}, y_{1}\right), \ldots,\left(t_{q}, x_{q}, y_{q}\right)\right)$ denote the transcript of the interaction of $\mathcal{A}$ with $\widetilde{P}$. For any two subsets $U$ and $V$ of $\{0,1\}^{n}$, let

$$
\mu(\widetilde{\mathcal{Q}}, U, V)=|\{((t, x, y), u, v) \in \widetilde{\mathcal{Q}} \times U \times V: x \oplus u=\Gamma(y \oplus v)\}| .
$$

Then, assuming $9 n \leq q \leq N / 2$, one has

$$
\underset{\widetilde{P}, \omega}{\operatorname{Pr}}\left[\exists U, V \subseteq\{0,1\}^{n}: \mu(\widetilde{\mathcal{Q}}, U, V) \geq \frac{q|U||V|}{N}+\frac{2 q^{2} \sqrt{|U||V|}}{N}+3 \sqrt{n q|U||V|}\right] \leq \frac{2}{N},
$$

where the probability is taken over the random choice of $\widetilde{P}$ and the random coins $\omega$ of $\mathcal{A}$. 


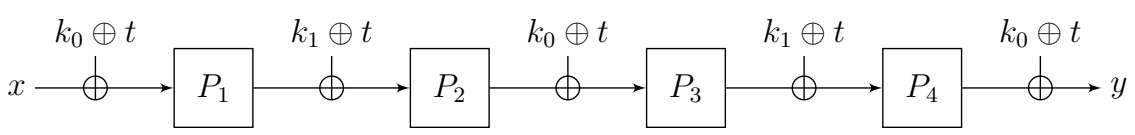

Fig. 2. The 4-round tweakable Even-Mansour construction with a $2 n$-bit key $\left(k_{0}, k_{1}\right)$ and an $n$-bit tweak $t$.

The proof of this lemma is a simple generalization of the one from $\left[\mathrm{CLL}^{+} 14\right]$ and can be found in Appendix A.

\section{Beyond-Birthday-Bound Security}

\subsection{Statement of the Result and Discussion}

In this section, we consider the 4-round tweakable Even-Mansour construction $\operatorname{TEM}[n, 4, \mathbf{f}]$ with $2 n$-bit keys and $n$-bit tweaks depicted on Figure 2 . The main result of this paper is the following one:

Theorem 1. Let $\mathbf{f}=\left(f_{0}, \ldots, f_{4}\right)$ where $f_{i}\left(\left(k_{0}, k_{1}\right), t\right)=k_{i \bmod 2} \oplus$ t. Let $q_{c}, q_{p}$ be two integers such that $9 n \leq q_{c}$ and $q_{p}+3 q_{c}+1 \leq N / 2$. Then one has

$$
\mathbf{A d v}_{\mathrm{TEM}[n, 4, \mathbf{f}]}^{\mathrm{cca}}\left(q_{c}, q_{p}\right) \leq \frac{44 q_{c}^{3 / 2}+38 q_{c} \sqrt{q_{p}}+(30+3 \sqrt{n}) q_{p} \sqrt{q_{c}}+4 q_{p}^{3 / 2}+2}{N} .
$$

Hence, this construction ensures CCA-security as long as $q_{c}$ and $q_{p}$ are small compared to $2^{2 n / 3}$, up to logarithmic terms in $N=2^{n}$.

The proof follows the H-coefficients method exposed in Section 2.3. In Section 3.2, we begin by describing the set of bad transcripts and upper bound the probability to get such a transcript in the ideal world. Then, for any good attainable transcript $\tau$, we prove in Section 3.3 that the ratio between the probability to get $\tau$ in the real world and in the ideal world is close enough to 1 .

\subsection{Definition and Probability of Bad Transcripts}

The first step is to define the set of bad transcripts. Let $\tau=\left(\mathcal{Q}_{C}, \mathcal{Q}_{P_{1}}, \ldots, \mathcal{Q}_{P_{4}},\left(k_{0}, k_{1}\right)\right)$ be an attainable transcript, with $\left|\mathcal{Q}_{C}\right|=q_{c}$ and $\left|\mathcal{Q}_{P_{i}}\right|=q_{p}$ for $i=1, \ldots, 4$. In all the following, we let, for $i \in\{1, \ldots, 4\}$,

$$
\begin{aligned}
U_{i} & =\left\{u_{i} \in\{0,1\}^{n}:\left(u_{i}, v_{i}\right) \in \mathcal{Q}_{P_{i}}\right\} \\
V_{i} & =\left\{v_{i} \in\{0,1\}^{n}:\left(u_{i}, v_{i}\right) \in \mathcal{Q}_{P_{i}}\right\}
\end{aligned}
$$

denote the domains and ranges of $\mathcal{Q}_{P_{i}}$ respectively. We also define three quantities characterizing the transcript,

$$
\begin{aligned}
\alpha_{1} & \stackrel{\text { def }}{=}\left|\left\{\left((t, x, y), u_{1}\right) \in \mathcal{Q}_{C} \times U_{1}: x \oplus k_{0} \oplus t=u_{1}\right\}\right| \\
\alpha_{4} & \stackrel{\text { def }}{=}\left|\left\{\left((t, x, y), v_{4}\right) \in \mathcal{Q}_{C} \times V_{4}: y \oplus k_{0} \oplus t=v_{4}\right\}\right| \\
\alpha_{2,3} & \stackrel{\text { def }}{=}\left|\left\{\left((t, x, y), v_{2}, u_{3}\right) \in \mathcal{Q}_{C} \times V_{2} \times U_{3}: v_{2} \oplus k_{0} \oplus t=u_{3}\right\}\right| .
\end{aligned}
$$


We also define two quantities depending respectively on $\mathcal{Q}_{P_{2}}$ and $\mathcal{Q}_{P_{3}}$ :

$$
\begin{aligned}
& \nu_{2} \stackrel{\text { def }}{=}\left|\left\{\left(\left(u_{2}, v_{2}\right),\left(u_{2}^{\prime}, v_{2}^{\prime}\right)\right) \in\left(\mathcal{Q}_{P_{2}}\right)^{2}:\left(u_{2}, v_{2}\right) \neq\left(u_{2}^{\prime}, v_{2}^{\prime}\right), u_{2} \oplus v_{2}=u_{2}^{\prime} \oplus v_{2}^{\prime}\right\}\right| \\
& \nu_{3} \stackrel{\text { def }}{=}\left|\left\{\left(\left(u_{3}, v_{3}\right),\left(u_{3}^{\prime}, v_{3}^{\prime}\right)\right) \in\left(\mathcal{Q}_{P_{3}}\right)^{2}:\left(u_{3}, v_{3}\right) \neq\left(u_{3}^{\prime}, v_{3}^{\prime}\right), u_{3} \oplus v_{3}=u_{3}^{\prime} \oplus v_{3}^{\prime}\right\}\right| .
\end{aligned}
$$

Definition 1. We say that a transcript $\tau$ is bad if at least one of the following conditions is fulfilled:

$(B-1)$ there exists $(t, x, y) \in \mathcal{Q}_{C},\left(u_{1}, v_{1}\right) \in \mathcal{Q}_{P_{1}}$, and $\left(u_{4}, v_{4}\right) \in \mathcal{Q}_{P_{4}}$ such that $k_{0} \oplus t=x \oplus u_{1}=$ $v_{4} \oplus y$

(B-2) there exists $(t, x, y) \in \mathcal{Q}_{C},\left(u_{1}, v_{1}\right) \in \mathcal{Q}_{P_{1}}$, and $\left(u_{2}, v_{2}\right) \in \mathcal{Q}_{P_{2}}$ such that $k_{0} \oplus t=x \oplus u_{1}$ and $k_{1} \oplus t=v_{1} \oplus u_{2}$;

(B-3) there exists $(t, x, y) \in \mathcal{Q}_{C},\left(u_{3}, v_{3}\right) \in \mathcal{Q}_{P_{3}}$, and $\left(u_{4}, v_{4}\right) \in \mathcal{Q}_{P_{4}}$ such that $k_{1} \oplus t=v_{3} \oplus u_{4}$ and $k_{0} \oplus t=v_{4} \oplus y$;

$(B-4) \alpha_{1} \geq \sqrt{q_{c}} / 2$

$(B-5) \quad \alpha_{4} \geq \sqrt{q_{c}} / 2$

(B-6) $\alpha_{2,3} \geq q_{p} \sqrt{q_{c}}$

(B-7) $\nu_{2} \geq \sqrt{q_{p}}$

$(B-8) \nu_{3} \geq \sqrt{q_{p}}$.

Otherwise we say that $\tau$ is good. ${ }^{5}$ We denote $\Theta_{\mathrm{good}}$, resp. $\Theta_{\mathrm{bad}}$ the set of good, resp. bad transcripts.

We start by upper bounding the probability of getting bad transcripts in the ideal world.

Lemma 3. Assume that $9 n \leq q_{c} \leq N / 2$ and $q_{p} \leq N / 2$. Then one has

$$
\operatorname{Pr}\left[T_{\mathrm{id}} \in \Theta_{\mathrm{bad}}\right] \leq \frac{2 q_{c}^{2} q_{p}+3 q_{c} q_{p}^{2}}{N^{2}}+\frac{(5+3 \sqrt{n}) \sqrt{q_{c}} q_{p}+4 q_{p}^{3 / 2}+2}{N} .
$$

Proof. We upper bound the probability of each condition in turn. We denote $\Theta_{i}$ the set of attainable transcripts satisfying condition $(\mathrm{B}-i)$. Recall that in the ideal world, the key $\left(k_{0}, k_{1}\right)$ is drawn independently from the queries transcript.

Condition (B-1). Let $\mathrm{BadK}_{1}$ be the set of keys $k_{0}$ such that there exists $(t, x, y) \in \mathcal{Q}_{C}$, $\left(u_{1}, v_{1}\right) \in \mathcal{Q}_{P_{1}}$, and $\left(u_{4}, v_{4}\right) \in \mathcal{Q}_{P_{4}}$ such that $k_{0} \oplus t=x \oplus u_{1}=y \oplus v_{4}$. Note that BadK only depends on the queries transcript, hence for any constant $C$ we have, since $k_{0}$ is uniformly random,

$$
\operatorname{Pr}\left[T_{\text {id }} \in \Theta_{1}\right] \leq \operatorname{Pr}\left[\widetilde{P}_{0} \leftarrow_{\$} \operatorname{TP}(\mathcal{T}, n), \mathbf{P} \leftarrow_{\$}(\mathrm{P}(n))^{4}:\left|\operatorname{BadK}_{1}\right|>C\right]+\frac{C}{N} .
$$

Moreover, if we let

$$
\left.\mu\left(\mathcal{Q}_{C}, U_{1}, V_{4}\right) \stackrel{\text { def }}{=} \mid\left\{\left((t, x, y), u_{1}, v_{4}\right) \in \mathcal{Q}_{C} \times U_{1} \times V_{4}: x \oplus u_{1}=y \oplus v_{4}\right)\right\} \mid,
$$

\footnotetext{
${ }^{5}$ We define conditions (B-4) and (B-5) using $\sqrt{q_{c}} / 2$ rather than $\sqrt{q_{c}}$ in order to be able later to directly apply a previous result by Cogliati et al. [CLS15].
} 
then one clearly has

$$
\left|\mathrm{BadK}_{1}\right| \leq \mu\left(\mathcal{Q}_{C}, U_{1}, V_{4}\right)
$$

Hence, we can use Lemma 2 in order to upper-bound $\mid$ BadK $_{1} \mid$ with overwhelming probability (we consider $\mathcal{D}$ with access to the inner permutations as a probabilistic algorithm $\mathcal{A}$ interacting with the tweakable permutation $\widetilde{P}_{0}$, resulting in the transcript $\mathcal{Q}_{C}$, and we let $\Gamma$ be the identity mapping). For

$$
C=\frac{q_{c} q_{p}^{2}}{N}+\frac{2 q_{c}^{2} q_{p}}{N}+3 q_{p} \sqrt{n q_{c}}
$$

we obtain that

$$
\operatorname{Pr}\left[\widetilde{P}_{0} \leftarrow_{\$} \operatorname{TP}(\mathcal{T}, n), \mathbf{P} \leftarrow_{\$}(\mathrm{P}(n))^{4}:\left|\mathrm{BadK}_{1}\right|>C\right] \leq \frac{2}{N}
$$

Using (5) gives

$$
\operatorname{Pr}\left[T_{\mathrm{id}} \in \Theta_{1}\right] \leq \frac{q_{c} q_{p}^{2}}{N^{2}}+\frac{2 q_{c}^{2} q_{p}}{N^{2}}+\frac{3 q_{p} \sqrt{n q_{c}}}{N}+\frac{2}{N} .
$$

Conditions (B-2) and (B-3). We consider (B-2). For each $(t, x, y) \in \mathcal{Q}_{C},\left(u_{1}, v_{1}\right) \in \mathcal{Q}_{P_{1}}$, and $\left(u_{2}, v_{2}\right) \in \mathcal{Q}_{P_{2}}$, the probability, over the random draw of $\left(k_{0}, k_{1}\right)$, that $k_{0} \oplus t=x \oplus u_{1}$ and $k_{1} \oplus t=v_{1} \oplus u_{2}$ is $1 / N^{2}$ since $\left(k_{0}, k_{1}\right)$ is uniform and independent from the queries transcript. Summing over the $q_{c} q_{p}^{2}$ possibilities for $(t, x, y),\left(u_{1}, v_{1}\right)$, and $\left(u_{2}, v_{2}\right)$ yields

$$
\operatorname{Pr}\left[T_{\text {id }} \in \Theta_{2}\right] \leq \frac{q_{c} q_{p}^{2}}{N^{2}} .
$$

Similarly,

$$
\operatorname{Pr}\left[T_{\text {id }} \in \Theta_{3}\right] \leq \frac{q_{c} q_{p}^{2}}{N^{2}} .
$$

Conditions (B-4) and (B-5). We consider (B-4). Seeing $\alpha_{1}$ as a random variable over the random draw of $\left(k_{0}, k_{1}\right)$, one has

$$
\mathbb{E}\left[\alpha_{1}\right]=\sum_{(t, x, y) \in \mathcal{Q}_{C}} \sum_{u_{1} \in U_{1}} \operatorname{Pr}\left[k_{0}=x \oplus u_{1} \oplus t\right] \leq \frac{q_{c} q_{p}}{N} .
$$

Then, using Markov's inequality,

$$
\operatorname{Pr}\left[T_{\text {id }} \in \Theta_{4}\right]=\operatorname{Pr}\left[\alpha_{1} \geq \frac{\sqrt{q_{c}}}{2}\right] \leq \frac{2 \mathbb{E}\left[\alpha_{1}\right]}{\sqrt{q_{c}}} \leq \frac{2 q_{p} \sqrt{q_{c}}}{N} .
$$

Similarly,

$$
\operatorname{Pr}\left[T_{\mathrm{id}} \in \Theta_{5}\right] \leq \frac{2 q_{p} \sqrt{q_{c}}}{N}
$$

Condition (B-6). Again, we see $\alpha_{2,3}$ as a random variable over the random draw of $k_{0}$. Then

$$
\mathbb{E}\left[\alpha_{2,3}\right]=\sum_{(t, x, y) \in \mathcal{Q}_{C}} \sum_{v_{2} \in V_{2}} \sum_{u_{3} \in U_{3}} \operatorname{Pr}\left[k_{0}=v_{2} \oplus u_{3} \oplus t\right] \leq \frac{q_{c} q_{p}^{2}}{N} .
$$

Then, using Markov's inequality,

$$
\operatorname{Pr}\left[T_{\mathrm{id}} \in \Theta_{6}\right]=\operatorname{Pr}\left[\alpha_{2,3} \geq q_{p} \sqrt{q_{c}}\right] \leq \frac{\mathbb{E}\left[\alpha_{2,3}\right]}{q_{p} \sqrt{q_{c}}} \leq \frac{q_{p} \sqrt{q_{c}}}{N} .
$$


Conditions (B-7) and (B-8). Consider (B-7). We see the distinguisher combined with $\widetilde{P}_{0}$ and the inner permutations $P_{1}, P_{3}$, and $P_{4}$ as a probabilistic algorithm $\mathcal{A}$ interacting with $P_{2}$, and we see $\nu_{2}$ as a random variable over the random choice of $P_{2}$ and the randomness of $\mathcal{A}$. One has

$$
\mathbb{E}\left[\nu_{2}\right]=\sum_{\substack{(i, j) \\ 1 \leq i \neq j \leq q_{c}}} \operatorname{Pr}\left[u_{2, i} \oplus v_{2, i}=u_{2, j} \oplus v_{2, j}\right],
$$

where the queries to $P_{2}$ are ordered as they are issued by $\mathcal{A}$. Consider the $i$-th and the $j$-th query, and assume $w \log$ that $i<j$. If the $j$-th is a direct query $u_{2, j}$, then $v_{2, j}$ is uniformly random in a set of size $N-j+1$. Similarly, if this is a inverse query $v_{2, j}$, then $u_{2, j}$ is uniformly random in a set of size $N-j+1$. In all cases, the probability that $u_{2, i} \oplus v_{2, i}=u_{2, j} \oplus v_{2, j}$ is at most $1 /\left(N-q_{p}\right)$. Hence,

$$
\mathbb{E}\left[\nu_{2}\right] \leq \frac{q_{p}\left(q_{p}-1\right)}{N-q_{p}} \leq \frac{2 q_{p}^{2}}{N}
$$

Using Markov's inequality,

$$
\operatorname{Pr}\left[T_{\mathrm{id}} \in \Theta_{7}\right]=\operatorname{Pr}\left[\nu_{2} \geq \sqrt{q_{p}}\right] \leq \frac{2 q_{p}^{3 / 2}}{N} .
$$

Similarly,

$$
\operatorname{Pr}\left[T_{\mathrm{id}} \in \Theta_{8}\right] \leq \frac{2 q_{p}^{3 / 2}}{N} .
$$

The result follows by a union bound over all cases.

\subsection{Analysis of Good Transcripts}

In this section, we fix a good transcript $\tau=\left(\mathcal{Q}_{C}, \mathcal{Q}_{P_{1}}, \ldots, \mathcal{Q}_{P_{4}},\left(k_{0}, k_{1}\right)\right)$. By (4), we have to lower bound

$$
\mathrm{p}(\tau) \stackrel{\text { def }}{=} \operatorname{Pr}\left[\mathbf{P} \leftarrow \$(\mathrm{P}(n))^{4}: \operatorname{TEM}_{k_{0}, k_{1}}^{\mathbf{P}} \vdash \mathcal{Q}_{C} \mid P_{1} \vdash \mathcal{Q}_{P_{1}} \wedge \ldots \wedge P_{4} \vdash \mathcal{Q}_{P_{4}}\right] .
$$

The proof will proceed in two steps: first, we will lower bound the probability that permutations $P_{1}$ and $P_{4}$ satisfy some conditions given in the definition below, and then, assuming $\left(P_{1}, P_{4}\right)$ is good, we will lower bound the probability, over the choice of $P_{2}$ and $P_{3}$, that $\operatorname{TEM}_{k_{0}, k_{1}}^{\mathrm{P}} \vdash \mathcal{Q}_{C}$. For this second step, we will directly appeal to a previous result by Cogliati et al. [CLS15].

We start by giving the conditions defining good pairs of permutations $\left(P_{1}, P_{4}\right)$. We stress that these conditions cannot be accommodated in the definition of bad transcripts since they depend on values of $P_{1}$ and $P_{4}$ which do not appear in the queries transcript, so that they cannot be defined from the transcript $\tau$ alone. We also warn the reader upfront that conditions (C-5) and (C-6) are "dummy" conditions that will easily be seen to be impossible to fulfill, yet will allow us to cleanly use the previous result of Cogliati et al. [CLS15].

Definition 2. A pair of permutations $\left(P_{1}, P_{4}\right)$ such that $P_{1} \vdash \mathcal{Q}_{P_{1}}$ and $P_{4} \vdash \mathcal{Q}_{P_{4}}$ is said bad if at least one of the following conditions is fulfilled (see Figure 3 for a diagram of the first ten conditions): 
(C-1) there exists $(t, x, y) \in \mathcal{Q}_{C}, u_{2} \in U_{2}$, and $v_{3} \in V_{3}$ such that

$$
\left\{\begin{array}{l}
P_{1}\left(x \oplus k_{0} \oplus t\right) \oplus k_{1} \oplus t=u_{2} \\
P_{4}^{-1}\left(y \oplus k_{0} \oplus t\right) \oplus k_{1} \oplus t=v_{3}
\end{array}\right.
$$

(C-2) there exists $(t, x, y) \in \mathcal{Q}_{C},\left(u_{2}, v_{2}\right) \in \mathcal{Q}_{P_{2}}$, and $u_{3} \in U_{3}$ such that

$$
\left\{\begin{array}{l}
P_{1}\left(x \oplus k_{0} \oplus t\right) \oplus k_{1} \oplus t=u_{2} \\
v_{2} \oplus k_{0} \oplus t=u_{3}
\end{array}\right.
$$

(C-3) there exists $(t, x, y) \in \mathcal{Q}_{C},\left(u_{3}, v_{3}\right) \in \mathcal{Q}_{P_{3}}$, and $v_{2} \in V_{2}$ such that

$$
\left\{\begin{array}{l}
P_{4}^{-1}\left(y \oplus k_{0} \oplus t\right) \oplus k_{1} \oplus t=v_{3} \\
u_{3} \oplus k_{0} \oplus t=v_{2}
\end{array}\right.
$$

$\left(C\right.$-4) there exists $(t, x, y),\left(t^{\prime}, x^{\prime}, y^{\prime}\right),\left(t^{\prime \prime}, x^{\prime \prime}, y^{\prime \prime}\right) \in \mathcal{Q}_{C}$ with $(t, x, y)$ distinct from $\left(t^{\prime}, x^{\prime}, y^{\prime}\right)$ and from $\left(t^{\prime \prime}, x^{\prime \prime}, y^{\prime \prime}\right)$ such that

$$
\left\{\begin{array}{l}
P_{1}\left(x \oplus k_{0} \oplus t\right) \oplus t=P_{1}\left(x^{\prime} \oplus k_{0} \oplus t^{\prime}\right) \oplus t^{\prime} \\
P_{4}^{-1}\left(y \oplus k_{0} \oplus t\right) \oplus t=P_{4}^{-1}\left(y^{\prime \prime} \oplus k_{0} \oplus t^{\prime \prime}\right) \oplus t^{\prime \prime}
\end{array}\right.
$$

$\left(C\right.$-5) there exists $(t, x, y,) \neq\left(t^{\prime}, x^{\prime}, y^{\prime}\right) \in \mathcal{Q}_{C}$ such that

$$
\left\{\begin{array}{l}
P_{1}\left(x \oplus k_{0} \oplus t\right) \oplus t=P_{1}\left(x^{\prime} \oplus k_{0} \oplus t^{\prime}\right) \oplus t^{\prime} \\
t=t^{\prime}
\end{array}\right.
$$

(C-6) there exists $(t, x, y,) \neq\left(t^{\prime}, x^{\prime}, y^{\prime}\right) \in \mathcal{Q}_{C}$ such that

$$
\left\{\begin{array}{l}
P_{4}^{-1}\left(y \oplus k_{0} \oplus t\right) \oplus t=P_{4}^{-1}\left(y^{\prime} \oplus k_{0} \oplus t^{\prime}\right) \oplus t^{\prime} \\
t=t^{\prime}
\end{array}\right.
$$

(C-7) there exists $(t, x, y) \neq\left(t^{\prime}, x^{\prime}, y^{\prime}\right) \in \mathcal{Q}_{C}$ and $u_{2} \in U_{2}$ such that

$$
\left\{\begin{array}{l}
P_{1}\left(x \oplus k_{0} \oplus t\right) \oplus k_{1} \oplus t=u_{2} \\
P_{4}^{-1}\left(y \oplus k_{0} \oplus t\right) \oplus t=P_{4}^{-1}\left(y^{\prime} \oplus k_{0} \oplus t^{\prime}\right) \oplus t^{\prime} ;
\end{array}\right.
$$

(C-8) there exists $(t, x, y) \neq\left(t^{\prime}, x^{\prime}, y^{\prime}\right) \in \mathcal{Q}_{C}$ and $v_{3} \in V_{3}$ such that

$$
\left\{\begin{array}{l}
P_{4}^{-1}\left(y \oplus k_{0} \oplus t\right) \oplus k_{1} \oplus t=v_{3} \\
P_{1}\left(x \oplus k_{0} \oplus t\right) \oplus t=P_{1}\left(x^{\prime} \oplus k_{0} \oplus t^{\prime}\right) \oplus t^{\prime}
\end{array}\right.
$$

(C-9) there exists $(t, x, y) \neq\left(t^{\prime}, x^{\prime}, y^{\prime}\right) \in \mathcal{Q}_{C}$ and $\left(u_{2}, v_{2}\right),\left(u_{2}^{\prime}, v_{2}^{\prime}\right) \in \mathcal{Q}_{P_{2}}$ such that

$$
\left\{\begin{array}{l}
P_{1}\left(x \oplus k_{0} \oplus t\right) \oplus k_{1} \oplus t=u_{2} \\
P_{1}\left(x^{\prime} \oplus k_{0} \oplus t^{\prime}\right) \oplus k_{1} \oplus t^{\prime}=u_{2}^{\prime} \\
v_{2} \oplus t=v_{2}^{\prime} \oplus t^{\prime}
\end{array}\right.
$$


$\left(C\right.$-10) there exists $(t, x, y) \neq\left(t^{\prime}, x^{\prime}, y^{\prime}\right) \in \mathcal{Q}_{C}$ and $\left(u_{3}, v_{3}\right),\left(u_{3}^{\prime}, v_{3}^{\prime}\right) \in \mathcal{Q}_{P_{3}}$ such that

$$
\left\{\begin{array}{l}
P_{4}^{-1}\left(y \oplus k_{0} \oplus t\right) \oplus k_{1} \oplus t=v_{3} \\
P_{4}^{-1}\left(y^{\prime} \oplus k_{0} \oplus t^{\prime}\right) \oplus k_{1} \oplus t^{\prime}=v_{3}^{\prime} \\
u_{3} \oplus t=u_{3}^{\prime} \oplus t^{\prime} ;
\end{array}\right.
$$

$(C-11) \alpha_{2} \geq \sqrt{q_{c}}$

$(C-12) \alpha_{3} \geq \sqrt{q_{c}}$

$(C-13) \beta_{2} \geq \sqrt{q_{c}}$

$(C-14) \beta_{3} \geq \sqrt{q_{c}}$

where

$$
\begin{gathered}
\alpha_{2} \stackrel{\text { def }}{=}\left|\left\{(t, x, y) \in \mathcal{Q}_{C}: P_{1}\left(x \oplus k_{0} \oplus t\right) \oplus k_{1} \oplus t \in U_{2}\right\}\right|, \\
\alpha_{3} \stackrel{\text { def }}{=}\left|\left\{(t, x, y) \in \mathcal{Q}_{C}: P_{4}^{-1}\left(y \oplus k_{0} \oplus t\right) \oplus k_{1} \oplus t \in V_{3}\right\}\right|, \\
\beta_{2} \stackrel{\text { def }}{=} \mid\left\{(t, x, y) \in \mathcal{Q}_{C}: \exists\left(t^{\prime}, x^{\prime}, y^{\prime}\right) \neq(t, x, y),\right. \\
\left.P_{1}\left(x \oplus k_{0} \oplus t\right) \oplus t=P_{1}\left(x^{\prime} \oplus k_{0} \oplus t^{\prime}\right) \oplus t^{\prime}\right\} \mid, \\
\beta_{3} \stackrel{\text { def }}{=} \mid\left\{(t, x, y) \in \mathcal{Q}_{C}: \exists\left(t^{\prime}, x^{\prime}, y^{\prime}\right) \neq(t, x, y),\right. \\
\left.P_{4}^{-1}\left(y \oplus k_{0} \oplus t\right) \oplus t=P_{4}^{-1}\left(y^{\prime} \oplus k_{0} \oplus t^{\prime}\right) \oplus t^{\prime}\right\} \mid .
\end{gathered}
$$

Otherwise we say that $\left(P_{1}, P_{4}\right)$ is good. We denote $\Pi_{\mathrm{good}}$, resp. $\Pi_{\mathrm{bad}}$ the set of good, resp. bad pairs of permutations $\left(P_{1}, P_{4}\right)$ such that $P_{1} \vdash \mathcal{Q}_{P_{1}}$ and $P_{4} \vdash \mathcal{Q}_{P_{4}}$.

In all the following, we denote $\Pi$ the set of pairs of permutations $\left(P_{1}, P_{4}\right)$ such that $P_{1} \vdash \mathcal{Q}_{P_{1}}$ and $P_{4} \vdash \mathcal{Q}_{P_{4}}$. The first step towards studying good transcripts will be to upper bound the probability that the pair $\left(P_{1}, P_{4}\right)$ is bad.

Lemma 4. For any integers $q_{c}$ and $q_{p}$ such that $q_{p}+q_{c}+1 \leq N / 2$, one has

$$
\operatorname{Pr}\left[\left(P_{1}, P_{4}\right) \in \Pi_{\mathrm{bad}}\right] \leq \frac{4 q_{c}^{3}+16 q_{c}^{2} q_{p}+4 q_{c} q_{p}^{2}}{N^{2}}+\frac{10 q_{c}^{3 / 2}+4 q_{c} \sqrt{q_{p}}+10 \sqrt{q_{c}} q_{p}}{N}
$$

where the probability is taken over the uniformly random draw of $\left(P_{1}, P_{4}\right)$ in $\Pi$.

Proof. We upper bound the probabilities of the fourteen conditions in turn. We denote $\Pi_{i}$ the set of pairs of permutations $\left(P_{1}, P_{4}\right) \in \Pi$ satisfying condition $(\mathrm{C}-i)$.

Condition (C-1). Fix $(t, x, y) \in \mathcal{Q}_{C}, u_{2} \in U_{2}$, and $v_{3} \in V_{3}$. Note that if $x \oplus k_{0} \oplus t=u_{1}$ for some $\left(u_{1}, v_{1}\right) \in \mathcal{Q}_{P_{1}}$, then $v_{1} \oplus k_{1} \oplus t$ cannot be equal to $u_{2}$ since otherwise $\tau$ would satisfy (B-2). Similarly, if $y \oplus k_{0} \oplus t=v_{4}$ for some $\left(u_{4}, v_{4}\right) \in \mathcal{Q}_{P_{4}}$, then $u_{4} \oplus k_{1} \oplus t$ cannot be equal to $v_{3}$ since otherwise $\tau$ would satisfy (B-3). On the other hand, if $x \oplus k_{0} \oplus t \notin U_{1}$ and $y \oplus k_{0} \oplus t \notin V_{4}$, then the probability over $\left(P_{1}, P_{4}\right) \leftarrow_{\$} \Pi$ that

$$
\left\{\begin{array}{l}
P_{1}\left(x \oplus k_{0} \oplus t\right)=u_{2} \oplus k_{1} \oplus t \\
P_{4}^{-1}\left(y \oplus k_{0} \oplus t\right)=v_{3} \oplus k_{1} \oplus t
\end{array}\right.
$$

is at most $1 /\left(N-q_{p}\right)^{2} \leq 4 / N^{2}$. (In more details, if $u_{2} \oplus k_{1} \oplus t \in V_{1}$ or $v_{3} \oplus k_{1} \oplus t \in U_{4}$, then this probability is zero, whereas otherwise it is exactly $1 /\left(N-q_{p}\right)^{2}$.) Summing over the at most $q_{c} q_{p}^{2}$ possibilities for $(t, x, y), u_{2}$, and $v_{3}$ yields

$$
\operatorname{Pr}\left[\left(P_{1}, P_{4}\right) \in \Pi_{1}\right] \leq \frac{4 q_{c} q_{p}^{2}}{N^{2}} .
$$


$(\mathrm{C}-1)$

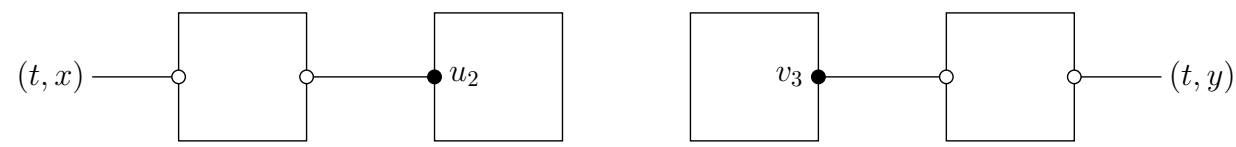

$(\mathrm{C}-2)$
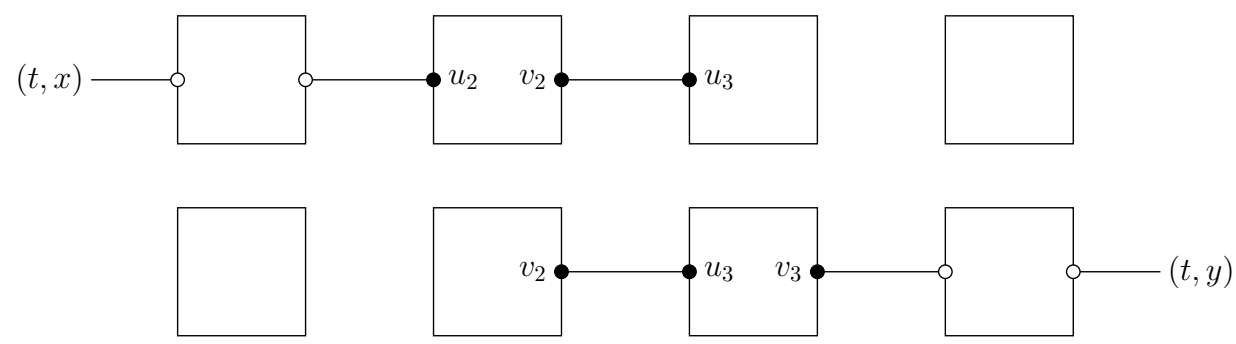

$(\mathrm{C}-4)$

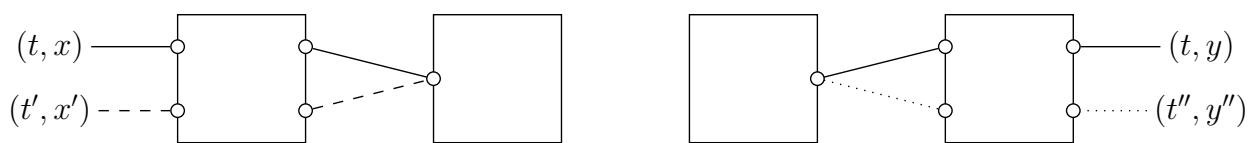

$(\mathrm{C}-5)$

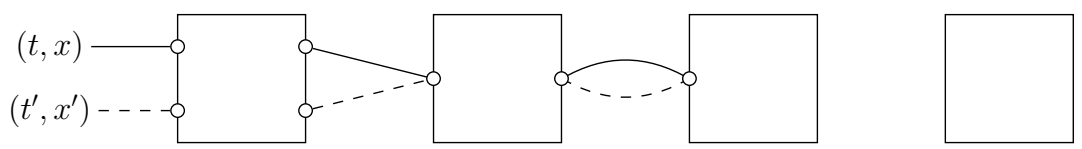

$(\mathrm{C}-6)$
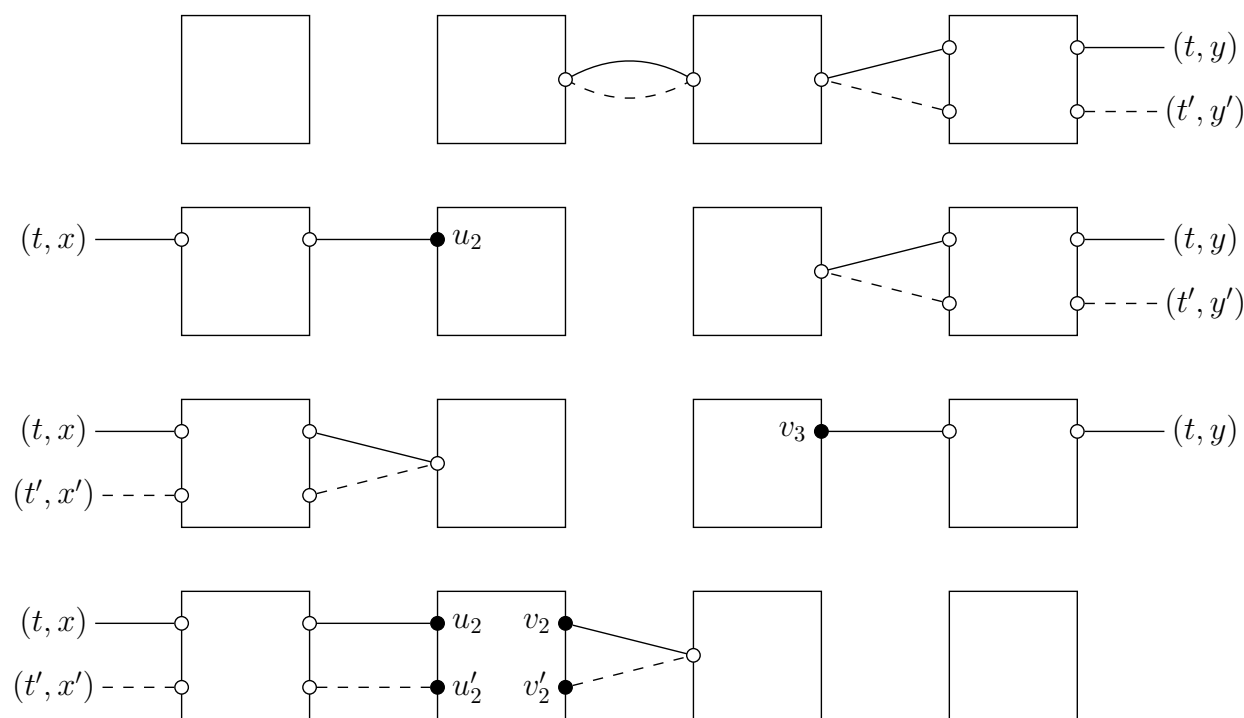

$(\mathrm{C}-10)$

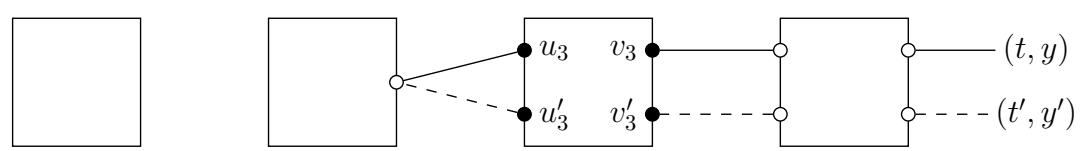

Fig. 3. The ten "collision" conditions characterizing a bad pair of permutations $\left(P_{1}, P_{4}\right)$. Black dots correspond to pairs $\left(u_{2}, v_{2}\right) \in \mathcal{Q}_{P_{2}}$ or $\left(u_{3}, v_{3}\right) \in \mathcal{Q}_{P_{3}}$. Note that for $(\mathrm{C}-4)$ one might have $\left(t^{\prime}, x^{\prime}\right)=\left(t^{\prime \prime}, x^{\prime \prime}\right)$, and for (C-9) (resp. (C-10)) one might have $x \oplus t=x^{\prime} \oplus t^{\prime}$ (resp. $y \oplus t=y^{\prime} \oplus t^{\prime}$ ). 
Conditions (C-2) and (C-3). We consider (C-2), the reasoning for (C-3) is similar. Fix $(t, x, y) \in \mathcal{Q}_{C},\left(u_{2}, v_{2}\right) \in \mathcal{Q}_{P_{2}}$, and $u_{3} \in U_{3}$. Note first that for (C-2) to be satisfied, one must have $v_{2} \oplus k_{0} \oplus t=u_{3}$, and there are by definition at most $\alpha_{2,3}$ triplets $\left((t, x, y), v_{2}, u_{3}\right)$ satisfying this equality. If $x \oplus k_{0} \oplus t=u_{1}$ for some $\left(u_{1}, v_{1}\right) \in \mathcal{Q}_{P_{1}}$, then $v_{1} \oplus k_{1} \oplus t$ cannot be equal to $u_{2}$ since otherwise $\tau$ would satisfy (B-2). On the other hand, if $x \oplus k_{0} \oplus t \notin U_{1}$, then the probability that $P_{1}\left(x \oplus k_{0} \oplus t\right)=u_{2} \oplus k_{1} \oplus t$ is at most $1 /\left(N-q_{p}\right) \leq 2 / N$ (it is zero if $u_{2} \oplus k_{1} \oplus t \in V_{1}$, and $1 /\left(N-q_{p}\right)$ otherwise $)$. Summing over the at most $\alpha_{2,3}$ possibilities for $(t, x, y),\left(u_{2}, v_{2}\right)$, and $u_{3}$, with $\alpha_{2,3} \leq q_{p} \sqrt{q_{c}}$ since otherwise $\tau$ would satisfy (B-6), we obtain

$$
\operatorname{Pr}\left[\left(P_{1}, P_{4}\right) \in \Pi_{2}\right] \leq \frac{2 q_{p} \sqrt{q_{c}}}{N} .
$$

Similarly,

$$
\operatorname{Pr}\left[\left(P_{1}, P_{4}\right) \in \Pi_{3}\right] \leq \frac{2 q_{p} \sqrt{q_{c}}}{N}
$$

Condition (C-4). Fix $(t, x, y),\left(t^{\prime}, x^{\prime}, y^{\prime}\right),\left(t^{\prime \prime}, x^{\prime \prime}, y^{\prime \prime}\right) \in \mathcal{Q}_{C}$ with $(t, x, y)$ distinct from $\left(t^{\prime}, x^{\prime}, y^{\prime}\right)$ and from $\left(t^{\prime \prime}, x^{\prime \prime}, y^{\prime \prime}\right)$. First, note that if $x \oplus k_{0} \oplus t=x^{\prime} \oplus k_{0} \oplus t^{\prime}$ or $y \oplus k_{0} \oplus t=y^{\prime \prime} \oplus k_{0} \oplus t^{\prime \prime}$, then (C-4) cannot be satisfied. Hence, we assume that none of these two equalities holds. We consider three cases. Assume first that $x \oplus k_{0} \oplus t=u_{1}$ for some $\left(u_{1}, v_{1}\right) \in \mathcal{Q}_{P_{1}}$. Note that there are at most $\alpha_{1}$ possibilities for $(t, x, y)$, and $\alpha_{1} \leq \sqrt{q_{c}} / 2$ since otherwise $\tau$ would satisfy (B-4). Moreover $y \oplus k_{0} \oplus t \notin V_{4}$ since otherwise $\tau$ would satisfy (B-1). Hence, the probability that

$$
P_{4}^{-1}\left(y \oplus k_{0} \oplus t\right) \oplus t=P_{4}^{-1}\left(y^{\prime \prime} \oplus k_{0} \oplus t^{\prime \prime}\right) \oplus t^{\prime \prime}
$$

is at most $1 /\left(N-q_{p}-1\right) \leq 2 / N$. (In more details, if $y^{\prime \prime} \oplus k_{0} \oplus t^{\prime \prime} \in V_{4}$, then this probability is either zero if $P_{4}^{-1}\left(y^{\prime \prime} \oplus k_{0} \oplus t^{\prime \prime}\right) \oplus t \oplus t^{\prime \prime} \in U_{4}$, or exactly $1 /\left(N-q_{p}\right)$ otherwise, whereas if $y^{\prime \prime} \oplus k_{0} \oplus t^{\prime \prime} \notin V_{4}$, then this probability is at most $1 /\left(N-q_{p}-1\right)$.) Summing over the at most $\sqrt{q_{c}} / 2 \times q_{c}$ possibilities for $(t, x, y)$ and $\left(t^{\prime \prime}, x^{\prime \prime}, y^{\prime \prime}\right)$, the probability of this first case is at most $q_{c}^{3 / 2} / N$. The second case where $y \oplus k_{0} \oplus t \in V_{4}$ is handled similarly. Finally, consider the case where $x \oplus k_{0} \oplus t \notin U_{1}$ and $y \oplus k_{0} \oplus t \notin V_{4}$. Then the probability that

$$
\left\{\begin{array}{l}
P_{1}\left(x \oplus k_{0} \oplus t\right) \oplus t=P_{1}\left(x^{\prime} \oplus k_{0} \oplus t^{\prime}\right) \oplus t^{\prime} \\
P_{4}^{-1}\left(y \oplus k_{0} \oplus t\right) \oplus t=P_{4}^{-1}\left(y^{\prime \prime} \oplus k_{0} \oplus t^{\prime \prime}\right) \oplus t^{\prime \prime}
\end{array}\right.
$$

is at most $1 /\left(N-q_{p}-1\right)^{2} \leq 4 / N^{2}$. Summing over the at most $q_{c}^{3}$ possibilities for $(t, x, y)$, $\left(t^{\prime}, x^{\prime}, y^{\prime}\right)$, and $\left(t^{\prime \prime}, x^{\prime \prime}, y^{\prime \prime}\right)$, the probability of this third case is at most $4 q_{c}^{3} / N^{2}$. Overall, we obtain

$$
\operatorname{Pr}\left[\left(P_{1}, P_{4}\right) \in \Pi_{4}\right] \leq \frac{4 q_{c}^{3}}{N^{2}}+\frac{2 q_{c}^{3 / 2}}{N} .
$$

Conditions (C-5) and (C-6). These conditions cannot be satisfied. Indeed, assume that there exits $(t, x, y) \neq\left(t^{\prime}, x^{\prime}, y^{\prime}\right) \in \mathcal{Q}_{C}$ satisfying (C-5). Since $t=t^{\prime}$, then $x \neq x^{\prime}$ by the assumption that the distinguisher never makes pointless queries. This obviously implies that $P_{1}\left(x \oplus k_{0} \oplus t\right) \oplus t \neq P_{1}\left(x^{\prime} \oplus k_{0} \oplus t^{\prime}\right) \oplus t^{\prime}$, a contradiction. The reasoning is similar for (C-6). Hence,

$$
\operatorname{Pr}\left[\left(P_{1}, P_{4}\right) \in \Pi_{5}\right]=\operatorname{Pr}\left[\left(P_{1}, P_{4}\right) \in \Pi_{6}\right]=0 .
$$


Conditions (C-7) and (C-8). We consider condition (C-7). Fix queries $(t, x, y) \neq\left(t^{\prime}, x^{\prime}, y^{\prime}\right) \in \mathcal{Q}_{C}$ and $u_{2} \in U_{2}$. We will consider two cases: first, the case where $y \oplus k_{0} \oplus t \in V_{4}$, and then the case where $y \oplus k_{0} \oplus t \notin V_{4}$. For both cases, note that if $x \oplus k_{0} \oplus t=u_{1}$ for some $\left(u_{1}, v_{1}\right) \in \mathcal{Q}_{P_{1}}$, then $v_{1} \oplus k_{1} \oplus t$ cannot be equal to $u_{2}$ since otherwise $\tau$ would satisfy (B-2). Hence, we can assume that $x \oplus k_{0} \oplus t \notin U_{1}$. It follows that the probability that

$$
P_{1}\left(x \oplus k_{0} \oplus t\right) \oplus k_{1} \oplus t=u_{2}
$$

is at most $1 /\left(N-q_{p}\right) \leq 2 / N$ (it is zero if $u_{2} \oplus k_{1} \oplus t \in V_{1}$, and $1 /\left(N-q_{p}\right)$ otherwise). Summing over the at most $\alpha_{4}$ queries $(t, x, y) \in \mathcal{Q}_{C}$ such that $y \oplus k_{0} \oplus t \in V_{4}$, with $\alpha_{4} \leq \sqrt{q_{c}} / 2$ since otherwise $\tau$ would satisfy (B-5), and the $q_{p}$ possibilities for $u_{2}$, we see that the first case happens with probability at most $q_{p} \sqrt{q_{c}} / N$. Assume now that $y \oplus k_{0} \oplus t \notin V_{4}$. Then the probability that

$$
P_{4}^{-1}\left(y \oplus k_{0} \oplus t\right) \oplus t=P_{4}^{-1}\left(y^{\prime} \oplus k_{0} \oplus t^{\prime}\right) \oplus t^{\prime}
$$

is at most $1 /\left(N-q_{p}-1\right) \leq 2 / N$. (In more details, if $y \oplus k_{0} \oplus t=y^{\prime} \oplus k_{0} \oplus t^{\prime}$, then it can easily be seen that it cannot hold, whereas if $y \oplus k_{0} \oplus t \neq y^{\prime} \oplus k_{0} \oplus t^{\prime}$, the equation holds with probability at most $1 /\left(N-q_{p}-1\right)$.) Summing over the at most $q_{c}^{2} q_{p}$ possibilities for $(t, x, y)$, $\left(t^{\prime}, x^{\prime}, y^{\prime}\right)$, and $u_{2}$, we see that the probability of the second case is at most $4 q_{c}^{2} q_{p} / N^{2}$. Overall,

$$
\operatorname{Pr}\left[\left(P_{1}, P_{4}\right) \in \Pi_{7}\right] \leq \frac{q_{p} \sqrt{q_{c}}}{N}+\frac{4 q_{c}^{2} q_{p}}{N^{2}} .
$$

Similarly, one has

$$
\operatorname{Pr}\left[\left(P_{1}, P_{4}\right) \in \Pi_{8}\right] \leq \frac{q_{p} \sqrt{q_{c}}}{N}+\frac{4 q_{c}^{2} q_{p}}{N^{2}} .
$$

Conditions (C-9) and (C-10). Consider condition (C-9). First note that, if the condition is satisfied, we have $x \oplus k_{0} \oplus t \notin U_{1}, x^{\prime} \oplus k_{0} \oplus t^{\prime} \notin U_{1}, u_{2} \oplus k_{1} \oplus t \notin V_{1}$ and $u_{2}^{\prime} \oplus k_{1} \oplus t^{\prime} \notin V_{1}$, otherwise (B-2) is fulfilled. Moreover, if $\left(u_{2}, v_{2}\right)=\left(u_{2}^{\prime}, v_{2}^{\prime}\right)$, then $t=t^{\prime}$, thus $x=x^{\prime}$, which is impossible. Hence we must have $\left(u_{2}, v_{2}\right) \neq\left(u_{2}^{\prime}, v_{2}^{\prime}\right)$. The condition can be divided into two conditions:

9.1 there exists $(t, x, y) \neq\left(t^{\prime}, x^{\prime}, y^{\prime}\right) \in \mathcal{Q}_{C}$ and $\left(u_{2}, v_{2}\right) \neq\left(u_{2}^{\prime}, v_{2}^{\prime}\right) \in \mathcal{Q}_{P_{2}}$ such that $x \oplus t=x^{\prime} \oplus t^{\prime}$, $P_{1}\left(x \oplus k_{0} \oplus t\right)=u_{2} \oplus k_{1} \oplus t$ and $P_{1}\left(x^{\prime} \oplus k_{0} \oplus t^{\prime}\right)=u_{2}^{\prime} \oplus k_{1} \oplus t^{\prime}$ and $v_{2} \oplus t=v_{2}^{\prime} \oplus t^{\prime} ;$

9.2 there exists $(t, x, y) \neq\left(t^{\prime}, x^{\prime}, y^{\prime}\right) \in \mathcal{Q}_{C}$ and $\left(u_{2}, v_{2}\right) \neq\left(u_{2}^{\prime}, v_{2}^{\prime}\right) \in \mathcal{Q}_{P_{2}}$ such that $x \oplus t \neq x^{\prime} \oplus t^{\prime}$, $P_{1}\left(x \oplus k_{0} \oplus t\right)=u_{2} \oplus k_{1} \oplus t$ and $P_{1}\left(x^{\prime} \oplus k_{0} \oplus t^{\prime}\right)=u_{2}^{\prime} \oplus k_{1} \oplus t^{\prime}$ and $v_{2} \oplus t=v_{2}^{\prime} \oplus t^{\prime}$.

In the first case, one has

$$
u_{2} \oplus k_{1} \oplus t=P_{1}\left(x \oplus k_{0} \oplus t\right)=P_{1}\left(x^{\prime} \oplus k_{0} \oplus t^{\prime}\right)=u_{2}^{\prime} \oplus k_{1} \oplus t^{\prime},
$$

thus $u_{2} \oplus u_{2}^{\prime}=t \oplus t^{\prime}=v_{2} \oplus v_{2}^{\prime}$. Hence the first condition implies the following one: there exists $(t, x, y) \in \mathcal{Q}_{C}$ and $\left(u_{2}, v_{2}\right) \neq\left(u_{2}^{\prime}, v_{2}^{\prime}\right) \in \mathcal{Q}_{P_{2}}$ such that $P_{1}\left(x \oplus k_{0} \oplus t\right)=u_{2} \oplus k_{1} \oplus t$ and $u_{2} \oplus u_{2}^{\prime}=v_{2} \oplus v_{2}^{\prime}$, with $x \oplus k_{0} \oplus t \notin U_{1}$ and $u_{2} \oplus k_{1} \oplus t \notin V_{1}$. Since $\nu_{2}<\sqrt{q_{p}}$, the number of suitable $u_{2} \in U_{2}$ is lower than $\sqrt{q_{p}}$, and the probability that this first condition is fulfilled is at most $\frac{q_{c} \sqrt{q_{p}}}{N-q_{p}} \leq \frac{2 q_{c} \sqrt{q_{p}}}{N}$. For the second condition, fix any queries $(t, x, y) \neq\left(t^{\prime}, x^{\prime}, y^{\prime}\right) \in \mathcal{Q}_{C}$ such that $x \oplus t \neq x^{\prime} \oplus t^{\prime}, x \oplus k_{0} \oplus t \notin U_{1}, x^{\prime} \oplus k_{0} \oplus t^{\prime} \notin U_{1}$ and $\left(u_{2}, v_{2}\right) \in \mathcal{Q}_{P_{2}}$. If $v_{2} \oplus t \oplus t^{\prime} \notin V_{2}$, the condition cannot be fulfilled. Otherwise let $\left(u_{2}^{\prime}, v_{2}^{\prime}\right) \in \mathcal{Q}_{P_{2}}$ be the unique query such that $v_{2} \oplus t=v_{2}^{\prime} \oplus t^{\prime}$. 
Then the probability that $P_{1}\left(x \oplus k_{0} \oplus t\right)=u_{2} \oplus k_{1} \oplus t$ and $P_{1}\left(x^{\prime} \oplus k_{0} \oplus t^{\prime}\right)=u_{2}^{\prime} \oplus k_{1} \oplus t^{\prime}$ is at most $\frac{1}{\left(N-q_{p}\right)\left(N-q_{p}-1\right)}$. Finally, by summing over every possible tuple of queries, and by taking into account the condition 9.1, one has

$$
\operatorname{Pr}\left[\left(P_{1}, P_{4}\right) \in \Pi_{9}\right] \leq \frac{2 q_{c} \sqrt{q_{p}}}{N}+\frac{4 q_{c}^{2} q_{p}}{N^{2}} .
$$

Similarly,

$$
\operatorname{Pr}\left[\left(P_{1}, P_{4}\right) \in \Pi_{10}\right] \leq \frac{2 q_{c} \sqrt{q_{p}}}{N}+\frac{4 q_{c}^{2} q_{p}}{N^{2}} .
$$

Conditions (C-11) and (C-12). We see $\alpha_{2}$ (resp. $\left.\alpha_{3}\right)$ as a random variable over the choice of $P_{1}$ (resp. $\left.P_{4}\right)$. Note that

$$
\begin{aligned}
\alpha_{2} & =\left|\left\{(t, x, y) \in \mathcal{Q}_{C}: P_{1}\left(x \oplus k_{0} \oplus t\right) \oplus k_{1} \oplus t \in U_{2}\right\}\right| \\
& =\left|\left\{(t, x, y) \in \mathcal{Q}_{C}: x \oplus k_{0} \oplus t \notin U_{1}, P_{1}\left(x \oplus k_{0} \oplus t\right) \oplus k_{1} \oplus t \in U_{2}\right\}\right|,
\end{aligned}
$$

because, if $x \oplus k_{0} \oplus t \in U_{1}$ and $P_{1}\left(x \oplus k_{0} \oplus t\right) \oplus k_{1} \oplus t \in U_{2}$, then (B-2) is fulfilled. We denote $\mathcal{Q}_{C, 1}$ the subset of queries $(t, x, y) \in \mathcal{Q}_{C}$ such that $x \oplus k_{0} \oplus t \notin U_{1}$. Then

$$
\begin{aligned}
\mathbb{E}\left[\alpha_{2}\right] & =\sum_{(t, x, y) \in \mathcal{Q}_{C, 1}} \sum_{u_{2} \in U_{2}} \operatorname{Pr}\left[P_{1}\left(x \oplus k_{0} \oplus t\right)=u_{2} \oplus k_{1} \oplus t\right] \\
& \leq \sum_{(t, x, y) \in \mathcal{Q}_{C, 1}} \sum_{u_{2} \in U_{2}} \frac{1}{N-q_{p}} \\
& \leq \frac{2 q_{c} q_{p}}{N} .
\end{aligned}
$$

Using Markov's inequality, we get

$$
\operatorname{Pr}\left[\left(P_{1}, P_{4}\right) \in \Pi_{11}\right] \leq \frac{2 q_{p} \sqrt{q_{c}}}{N} .
$$

Similarly,

$$
\operatorname{Pr}\left[\left(P_{1}, P_{4}\right) \in \Pi_{12}\right] \leq \frac{2 q_{p} \sqrt{q_{c}}}{N} .
$$

Conditions (C-13) and (C-14). Consider condition (C-13). Note that

$$
\begin{gathered}
\beta_{2}=\mid\left\{(t, x, y) \in \mathcal{Q}_{C}: \exists\left(t^{\prime}, x^{\prime}, y^{\prime}\right) \neq(t, x, y)\right. \\
\left.P_{1}\left(x \oplus k_{0} \oplus t\right) \oplus t=P_{1}\left(x^{\prime} \oplus k_{0} \oplus t^{\prime}\right) \oplus t^{\prime}\right\} \mid \\
\leq \alpha_{1}+\mid\left\{(t, x, y) \in \mathcal{Q}_{C}: x \oplus k_{0} \oplus t \notin U_{1} \text { and } \exists\left(t^{\prime}, x^{\prime}, y^{\prime}\right) \neq(t, x, y),\right. \\
\left.P_{1}\left(x \oplus k_{0} \oplus t\right) \oplus t=P_{1}\left(x^{\prime} \oplus k_{0} \oplus t^{\prime}\right) \oplus t^{\prime}\right\} \mid
\end{gathered}
$$

We denote $\beta_{2}^{\prime}$ the last term of this sum. Thus

$$
\begin{aligned}
\mathbb{E}\left[\beta_{2}^{\prime}\right] & =\sum_{(t, x, y) \in \mathcal{Q}_{C, 1}} \sum_{\left(t^{\prime}, x^{\prime}, y^{\prime}\right) \neq(t, x, y)} \operatorname{Pr}\left[P_{1}\left(x \oplus k_{0} \oplus t\right) \oplus t=P_{1}\left(x^{\prime} \oplus k_{0} \oplus t^{\prime}\right) \oplus t^{\prime}\right] \\
& \leq \frac{q_{c}^{2}}{N-q_{p}-1} \leq \frac{2 q_{c}^{2}}{N}
\end{aligned}
$$


This inequality holds because, if $x \oplus t=x^{\prime} \oplus t^{\prime}$, then $t \neq t^{\prime}$ since the distinguisher never makes pointless queries, thus $P_{1}\left(x \oplus k_{0} \oplus t\right) \oplus t=P_{1}\left(x^{\prime} \oplus k_{0} \oplus t^{\prime}\right) \oplus t^{\prime}$ cannot be fulfilled. Otherwise,

$$
\operatorname{Pr}\left[P_{1}\left(x \oplus k_{0} \oplus t\right) \oplus t=P_{1}\left(x^{\prime} \oplus k_{0} \oplus t^{\prime}\right) \oplus t^{\prime}\right] \leq \frac{1}{N-q_{p}-1} .
$$

Finally, since (B-4) is not fulfilled, $\alpha_{1}<\sqrt{q_{c}} / 2$. Thus $\beta_{2} \geq \sqrt{q_{c}}$ implies $\beta_{2}^{\prime} \geq \sqrt{q_{c}} / 2$. Hence, using Markov's inequality,

$$
\operatorname{Pr}\left[\left(P_{1}, P_{4}\right) \in \Pi_{13}\right] \leq \operatorname{Pr}\left[\beta_{2}^{\prime} \geq \sqrt{q_{c}} / 2\right] \leq \frac{2 \mathbb{E}\left[\beta_{2}^{\prime}\right]}{\sqrt{q_{c}}} \leq \frac{4 q_{c}^{3 / 2}}{N} .
$$

Similarly,

$$
\operatorname{Pr}\left[\left(P_{1}, P_{4}\right) \in \Pi_{14}\right] \leq \frac{4 q_{c}^{3 / 2}}{N} .
$$

The result follows by an union bound over all conditions.

We are now ready for the second step of the reasoning.

Definition 3. Fix any pair of permutations $\left(P_{1}, P_{4}\right)$ such that $P_{1} \vdash \mathcal{Q}_{P_{1}}$ and $P_{4} \vdash \mathcal{Q}_{P_{4}}$. We define a new query transcript $\mathcal{Q}_{C}^{\prime}$ depending on $\left(P_{1}, P_{4}\right)$ as

$$
\mathcal{Q}_{C}^{\prime}=\left\{\left(t, P_{1}\left(x \oplus k_{0} \oplus t\right), P_{4}^{-1}\left(y \oplus k_{0} \oplus t\right)\right):(t, x, y) \in \mathcal{Q}_{C}\right\} .
$$

We also denote

$$
\tilde{\mathbf{p}}\left(\tau, P_{1}, P_{4}\right)=\operatorname{Pr}\left[P_{2}, P_{3} \leftarrow{ }_{\$} \mathrm{P}(n): \operatorname{TEM}_{k_{1}, k_{0}}^{P_{2}, P_{3}} \vdash \mathcal{Q}_{C}^{\prime} \mid\left(P_{2} \vdash \mathcal{Q}_{P_{2}}\right) \wedge\left(P_{3} \vdash \mathcal{Q}_{P_{3}}\right)\right] .
$$

Lemma 5. One has

$$
\frac{\operatorname{Pr}\left[T_{\mathrm{re}}=\tau\right]}{\operatorname{Pr}\left[T_{\mathrm{id}}=\tau\right]} \geq \sum_{\left(P_{1}, P_{4}\right) \in \Pi_{\text {good }}} \frac{\tilde{\mathrm{p}}\left(\tau, P_{1}, P_{4}\right)}{\left(\left(N-q_{p}\right) !\right)^{2} \prod_{i=1}^{m} 1 /(N)_{q_{i}}} .
$$

Proof. Clearly, once $P_{1}$ and $P_{4}$ are fixed, $\operatorname{TEM}_{k_{0}, k_{1}}^{P_{1}, P_{2}, P_{3}, P_{4}} \vdash \mathcal{Q}_{C}$ is equivalent to $\operatorname{TEM}_{k_{1}, k_{0}}^{P_{2}, P_{3}} \vdash \mathcal{Q}_{C}^{\prime}$. Hence,

$$
\begin{aligned}
\mathrm{p}(\tau) & =\sum_{\left(\bar{P}_{1}, \bar{P}_{4}\right) \in \Pi} \operatorname{Pr}\left[\left(P_{1}, P_{4}\right) \leftarrow_{\$} \Pi:\left(P_{1}=\bar{P}_{1}\right) \wedge\left(P_{4}=\bar{P}_{4}\right)\right] \tilde{\mathrm{p}}\left(\tau, \bar{P}_{1}, \bar{P}_{4}\right) \\
& \geq \sum_{\left(\bar{P}_{1}, \bar{P}_{4}\right) \in \Pi_{\text {good }}} \frac{\tilde{\mathrm{p}}\left(\tau, \bar{P}_{1}, \bar{P}_{4}\right)}{\left(\left(N-q_{p}\right) !\right)^{2}} .
\end{aligned}
$$

The result follows from Eq. (4).

We can now directly appeal to a previous result by Cogliati et al. [CLS15].

Lemma 6. Let $q_{c}$ and $q_{p}$ be two positive integers such that $q_{p}+3 q_{c} \leq N / 2$. Fix any pair of permutations $\left(P_{1}, P_{4}\right) \in \Pi_{\text {good }}$. Then

$$
\frac{\tilde{\mathrm{p}}\left(\tau, P_{1}, P_{4}\right)}{\prod_{i=1}^{m} 1 /(N)_{q_{i}}} \geq 1-\left(\frac{4 q_{c}\left(q_{p}+2 q_{c}\right)^{2}}{N^{2}}+\frac{14 q_{c}^{3 / 2}+4 \sqrt{q_{c}} q_{p}}{N}\right) .
$$


Proof. One can check that the queries transcript $\tau^{\prime}=\left(\mathcal{Q}_{C}^{\prime}, \mathcal{Q}_{P_{2}}, \mathcal{Q}_{P_{3}}\right)$ satisfies exactly the conditions defining a good transcript as per [CLS15, Definition 2]. Moreover, the ratio $\tilde{\mathbf{p}}\left(\tau, P_{1}, P_{4}\right) / \prod_{i=1}^{m} 1 /(N)_{q_{i}}$ is exactly the ratio of the probabilities to get $\tau^{\prime}$ in the real and in the ideal world once a good pair $\left(P_{1}, P_{4}\right)$ is fixed. Hence, we can apply [CLS15, Lemma 6] that directly yields the result. ${ }^{6}$

We are now ready to prove the main lemma of this section.

Lemma 7. Let $q_{c}$ and $q_{p}$ be two positive integers such that $q_{p}+3 q_{c}+1 \leq N / 2$. One has

$$
\frac{\operatorname{Pr}\left[T_{\mathrm{re}}=\tau\right]}{\operatorname{Pr}\left[T_{\mathrm{id}}=\tau\right]} \geq 1-\frac{20 q_{c}^{3}+32 q_{c}^{2} q_{p}+8 q_{c} q_{p}^{2}}{N^{2}}-\frac{24 q_{c}^{3 / 2}+4 q_{c} \sqrt{q_{p}}+14 \sqrt{q_{c}} q_{p}}{N} .
$$

Proof. From Lemmas 5 and 6, one has

$$
\begin{aligned}
\frac{\operatorname{Pr}\left[T_{\mathrm{re}}=\tau\right]}{\operatorname{Pr}\left[T_{\mathrm{id}}=\tau\right]} & \geq \sum_{\left(P_{1}, P_{4}\right) \in \Pi_{\mathrm{good}}} \frac{\tilde{\mathrm{p}}\left(\tau, P_{1}, P_{4}\right)}{\left(\left(N-q_{p}\right) !\right)^{2} \prod_{i=1}^{m} 1 /(N)_{q_{i}}} \\
& \geq\left(1-\frac{4 q_{c}\left(q_{p}+2 q_{c}\right)^{2}}{N^{2}}-\frac{14 q_{c}^{3 / 2}+4 \sqrt{q_{c}} q_{p}}{N}\right) \sum_{\Pi_{\text {good }}} \frac{1}{\left(\left(N-q_{p}\right) !\right)^{2}} \\
& =\left(1-\frac{4 q_{c}\left(q_{p}+2 q_{c}\right)^{2}}{N^{2}}-\frac{14 q_{c}^{3 / 2}+4 \sqrt{q_{c}} q_{p}}{N}\right) \frac{\left|\Pi_{\text {good }}\right|}{\left(\left(N-q_{p}\right) !\right)^{2}} \\
& =\left(1-\frac{4 q_{c}\left(q_{p}+2 q_{c}\right)^{2}}{N^{2}}-\frac{14 q_{c}^{3 / 2}+4 \sqrt{q_{c}} q_{p}}{N}\right) \operatorname{Pr}\left[\left(P_{1}, P_{4}\right) \in \Pi_{\text {good }}\right],
\end{aligned}
$$

where the last probability is taken over the random draw of $\left(P_{1}, P_{4}\right)$ from $\Pi$, the set of pairs of permutations satisfying $P_{1} \vdash \mathcal{Q}_{P_{1}}$ and $P_{4} \vdash \mathcal{Q}_{P_{4}}$. Using Lemma 4 , one has

$$
\begin{aligned}
\frac{\operatorname{Pr}\left[T_{\mathrm{re}}=\tau\right]}{\operatorname{Pr}\left[T_{\mathrm{id}}=\tau\right]} \geq & \left(1-\frac{4 q_{c}^{3}+16 q_{c}^{2} q_{p}+4 q_{c} q_{p}^{2}}{N^{2}}-\frac{10 q_{c}^{3 / 2}+4 q_{c} \sqrt{q_{p}}+10 \sqrt{q_{c}} q_{p}}{N}\right) \\
& \times\left(1-\frac{4 q_{c}\left(q_{p}+2 q_{c}\right)^{2}}{N^{2}}-\frac{14 q_{c}^{3 / 2}+4 \sqrt{q_{c}} q_{p}}{N}\right) \\
\geq & 1-\frac{20 q_{c}^{3}+32 q_{c}^{2} q_{p}+8 q_{c} q_{p}^{2}}{N^{2}}-\frac{24 q_{c}^{3 / 2}+4 q_{c} \sqrt{q_{p}}+14 \sqrt{q_{c}} q_{p}}{N} .
\end{aligned}
$$

Concluding. We are now ready to prove Theorem 1 . Combining Lemmas 1,3 , and 7 , one has

$$
\begin{aligned}
\operatorname{Adv}_{\text {TEM }[n, 4, \mathbf{f}]}^{\text {cca }}\left(q_{c}, q_{p}\right) \leq & \frac{2 q_{c}^{2} q_{p}+3 q_{c} q_{p}^{2}}{N^{2}}+\frac{(5+3 \sqrt{n}) \sqrt{q_{c}} q_{p}+4 q_{p}^{3 / 2}+2}{N} \\
& +\frac{20 q_{c}^{3}+32 q_{c}^{2} q_{p}+8 q_{c} q_{p}^{2}}{N^{2}}+\frac{24 q_{c}^{3 / 2}+4 q_{c} \sqrt{q_{p}}+14 \sqrt{q_{c}} q_{p}}{N}
\end{aligned}
$$

\footnotetext{
${ }^{6}$ Even though this might not be apparent to the reader unfamiliar with [CLS15], the proof of Lemma 6 in that paper does not rely on the xor-universal hash functions $h_{1}$ and $h_{2}$ appearing in the definition of good transcripts of [CLS15].
} 


$$
\begin{aligned}
\leq & \frac{20 q_{c}^{3}+34 q_{c}^{2} q_{p}+11 q_{c} q_{p}^{2}}{N^{2}} \\
& +\frac{24 q_{c}^{3 / 2}+4 q_{c} \sqrt{q_{p}}+(19+3 \sqrt{n}) \sqrt{q_{c}} q_{p}+4 q_{p}^{3 / 2}+2}{N} .
\end{aligned}
$$

Since the result holds trivially when $q_{c}^{3}>N^{2}, q_{c}^{2} q_{p}>N^{2}$, or $q_{c} q_{p}^{2}>N^{2}$, we can assume that $q_{c}^{3} \leq N^{2}, q_{c}^{2} q_{p} \leq N^{2}$, and $q_{c} q_{p}^{2} \leq N^{2}$, so that

$$
\frac{q_{c}^{3}}{N^{2}} \leq \frac{q_{c}^{3 / 2}}{N}, \quad \frac{q_{c}^{2} q_{p}}{N^{2}} \leq \frac{q_{c} \sqrt{q_{p}}}{N}, \quad \text { and } \quad \frac{q_{c} q_{p}^{2}}{N^{2}} \leq \frac{\sqrt{q_{c}} q_{p}}{N}
$$

Thus

$$
\operatorname{Adv}_{\mathrm{TEM}[n, 4, \mathbf{f}]}^{\mathrm{cca}}\left(q_{c}, q_{p}\right) \leq \frac{44 q_{c}^{3 / 2}+38 q_{c} \sqrt{q_{p}}+(30+3 \sqrt{n}) q_{p} \sqrt{q_{c}}+4 q_{p}^{3 / 2}+2}{N},
$$

which concludes the proof of Theorem 1.

\section{Acknowledgment}

We wish to thank the anonymous reviewers of ASIACRYPT 2015 for their useful suggestions.

\section{References}

$\left[\mathrm{ABL}^{+} 13\right]$ Elena Andreeva, Andrey Bogdanov, Atul Luykx, Bart Mennink, Elmar Tischhauser, and Kan Yasuda. Parallelizable and Authenticated Online Ciphers. In Kazue Sako and Palash Sarkar, editors, Advances in Cryptology - ASIACRYPT 2013 - Proceedings, Part I, volume 8269 of LNCS, pages 424-443. Springer, 2013.

[BK03] Mihir Bellare and Tadayoshi Kohno. A Theoretical Treatment of Related-Key Attacks: RKA-PRPs, RKA-PRFs, and Applications. In Eli Biham, editor, Advances in Cryptology - EUROCRYPT 2003, volume 2656 of $L N C S$, pages 491-506. Springer, 2003.

$\left[\mathrm{BKL}^{+} 12\right]$ Andrey Bogdanov, Lars R. Knudsen, Gregor Leander, François-Xavier Standaert, John P. Steinberger, and Elmar Tischhauser. Key-Alternating Ciphers in a Provable Setting: Encryption Using a Small Number of Public Permutations - (Extended Abstract). In David Pointcheval and Thomas Johansson, editors, Advances in Cryptology - EUROCRYPT 2012, volume 7237 of LNCS, pages 45-62. Springer, 2012.

$\left[\mathrm{CLL}^{+} 14\right]$ Shan Chen, Rodolphe Lampe, Jooyoung Lee, Yannick Seurin, and John P. Steinberger. Minimizing the Two-Round Even-Mansour Cipher. In Juan A. Garay and Rosario Gennaro, editors, Advances in Cryptology - CRYPTO 2014 (Proceedings, Part I), volume 8616 of LNCS, pages 39-56. Springer, 2014. Full version available at http://eprint.iacr.org/2014/443.

[CLS15] Benoît Cogliati, Rodolphe Lampe, and Yannick Seurin. Tweaking Even-Mansour Ciphers. In Rosario Gennaro and Matthew Robshaw, editors, Advances in Cryptology - CRYPTO 2015 Proceedings, Part I, volume 9215 of $L N C S$, pages 189-208. Springer, 2015. Full version available at http://eprint.iacr.org/2015/539.

[Cro00] Paul Crowley. Mercy: A Fast Large Block Cipher for Disk Sector Encryption. In Bruce Schneier, editor, Fast Software Encryption - FSE 2000, volume 1978 of LNCS, pages 49-63. Springer, 2000.

[CS06] Debrup Chakraborty and Palash Sarkar. A General Construction of Tweakable Block Ciphers and Different Modes of Operations. In Helger Lipmaa, Moti Yung, and Dongdai Lin, editors, Information Security and Cryptology - Inscrypt 2006, volume 4318 of LNCS, pages 88-102. Springer, 2006.

[CS14] Shan Chen and John Steinberger. Tight Security Bounds for Key-Alternating Ciphers. In Phong Q. Nguyen and Elisabeth Oswald, editors, Advances in Cryptology - EUROCRYPT 2014, volume 8441 of $L N C S$, pages 327-350. Springer, 2014. Full version available at http://eprint.iacr.org/ 2013/222. 
[CS15] Benoît Cogliati and Yannick Seurin. On the Provable Security of the Iterated Even-Mansour Cipher against Related-Key and Chosen-Key Attacks. In Elisabeth Oswald and Marc Fischlin, editors, Advances in Cryptology - EUROCRYPT 2015 - Proceedings, Part I, volume 9056 of LNCS, pages 584-613. Springer, 2015. Full version available at http://eprint.iacr.org/2015/069.

[DDKS14] Itai Dinur, Orr Dunkelman, Nathan Keller, and Adi Shamir. Cryptanalysis of Iterated EvenMansour Schemes with Two Keys. In Palash Sarkar and Tetsu Iwata, editors, Advances in Cryptology - ASIACRYPT 2014 (Proceedings, Part I), volume 8873 of LNCS, pages 439-457. Springer, 2014. Full version available at http://eprint.iacr.org/2013/674.

[DR01] Joan Daemen and Vincent Rijmen. The Wide Trail Design Strategy. In Bahram Honary, editor, Cryptography and Coding 2001, volume 2260 of LNCS, pages 222-238. Springer, 2001.

[EM97] Shimon Even and Yishay Mansour. A Construction of a Cipher from a Single Pseudorandom Permutation. Journal of Cryptology, 10(3):151-162, 1997.

$\left[\mathrm{FLS}^{+} 10\right] \quad$ Niels Ferguson, Stefan Lucks, Bruce Schneier, Doug Whiting, Mihir Bellare, Tadayoshi Kohno, Jon Callas, and Jesse Walker. The Skein Hash Function Family. SHA3 Submission to NIST (Round 3), 2010.

[FP15] Pooya Farshim and Gordon Procter. The Related-Key Security of Iterated Even-Mansour Ciphers. In Gregor Leander, editor, Fast Software Encryption - FSE 2015, volume 9054 of LNCS, pages 342-363. Springer, 2015. Full version available at http://eprint.iacr.org/2014/953.

$\left[\mathrm{GHL}^{+} 07\right]$ David Goldenberg, Susan Hohenberger, Moses Liskov, Elizabeth Crump Schwartz, and Hakan Seyalioglu. On Tweaking Luby-Rackoff Blockciphers. In Kaoru Kurosawa, editor, Advances in Cryptology - ASIACRYPT 200\%, volume 4833 of LNCS, pages 342-356. Springer, 2007.

[GPPR11] Jian Guo, Thomas Peyrin, Axel Poschmann, and Matthew J. B. Robshaw. The LED Block Cipher. In Bart Preneel and Tsuyoshi Takagi, editors, Cryptographic Hardware and Embedded Systems CHES 2011, volume 6917 of LNCS, pages 326-341. Springer, 2011.

[HR03] Shai Halevi and Phillip Rogaway. A Tweakable Enciphering Mode. In Dan Boneh, editor, Advances in Cryptology - CRYPTO 2003, volume 2729 of LNCS, pages 482-499. Springer, 2003.

[HR04] Shai Halevi and Phillip Rogaway. A Parallelizable Enciphering Mode. In Tatsuaki Okamoto, editor, Topics in Cryptology - CT-RSA 2004, volume 2964 of LNCS, pages 292-304. Springer, 2004 .

[JNP14] Jérémy Jean, Ivica Nikolic, and Thomas Peyrin. Tweaks and Keys for Block Ciphers: The TWEAKEY Framework. In Palash Sarkar and Tetsu Iwata, editors, Advances in Cryptology ASIACRYPT 2014 - Proceedings, Part II, volume 8874 of LNCS, pages 274-288. Springer, 2014.

[LPS12] Rodolphe Lampe, Jacques Patarin, and Yannick Seurin. An Asymptotically Tight Security Analysis of the Iterated Even-Mansour Cipher. In Xiaoyun Wang and Kazue Sako, editors, Advances in Cryptology - ASIACRYPT 2012, volume 7658 of LNCS, pages 278-295. Springer, 2012.

[LRW02] Moses Liskov, Ronald L. Rivest, and David Wagner. Tweakable Block Ciphers. In Moti Yung, editor, Advances in Cryptology - CRYPTO 2002, volume 2442 of LNCS, pages 31-46. Springer, 2002.

[LS14] Rodolphe Lampe and Yannick Seurin. Security Analysis of Key-Alternating Feistel Ciphers. In Carlos Cid and Christian Rechberger, editors, Fast Software Encryption - FSE 2014, volume 8540 of $L N C S$, pages 243-264. Springer, 2014.

[LST12] Will Landecker, Thomas Shrimpton, and R. Seth Terashima. Tweakable Blockciphers with Beyond Birthday-Bound Security. In Reihaneh Safavi-Naini and Ran Canetti, editors, Advances in Cryptology - CRYPTO 2012, volume 7417 of $L N C S$, pages 14-30. Springer, 2012. Full version available at http://eprint.iacr.org/2012/450.

[Men15] Bart Mennink. Optimally Secure Tweakable Blockciphers. In Gregor Leander, editor, Fast Software Encryption - FSE 2015, volume 9054 of LNCS, pages 428-448. Springer, 2015. Full version available at http://eprint.iacr.org/2015/363.

[MI08] Atsushi Mitsuda and Tetsu Iwata. Tweakable Pseudorandom Permutation from Generalized Feistel Structure. In Joonsang Baek, Feng Bao, Kefei Chen, and Xuejia Lai, editors, ProvSec 2008, volume 5324 of $L N C S$, pages 22-37. Springer, 2008.

[Min06] Kazuhiko Minematsu. Improved Security Analysis of XEX and LRW Modes. In Eli Biham and Amr M. Youssef, editors, Selected Areas in Cryptography - SAC 2006, volume 4356 of LNCS, pages 96-113. Springer, 2006.

[Min09] Kazuhiko Minematsu. Beyond-Birthday-Bound Security Based on Tweakable Block Cipher. In Orr Dunkelman, editor, Fast Software Encryption - FSE 2009, volume 5665 of LNCS, pages 308-326. Springer, 2009. 
[Pat08] Jacques Patarin. The "Coefficients H" Technique. In Roberto Maria Avanzi, Liam Keliher, and Francesco Sica, editors, Selected Areas in Cryptography - SAC 2008, volume 5381 of LNCS, pages 328-345. Springer, 2008.

[Pro14] Gordon Procter. A Note on the CLRW2 Tweakable Block Cipher Construction. IACR Cryptology ePrint Archive, Report 2014/111, 2014. Available at http://eprint.iacr.org/2014/111.

[RBB03] Phillip Rogaway, Mihir Bellare, and John Black. OCB: A block-cipher mode of operation for efficient authenticated encryption. ACM Trans. Inf. Syst. Secur., 6(3):365-403, 2003.

[Rog04] Phillip Rogaway. Efficient Instantiations of Tweakable Blockciphers and Refinements to Modes OCB and PMAC. In Pil Joong Lee, editor, Advances in Cryptology - ASIACRYPT 2004, volume 3329 of LNCS, pages 16-31. Springer, 2004.

[RZ11] Phillip Rogaway and Haibin Zhang. Online Ciphers from Tweakable Blockciphers. In Aggelos Kiayias, editor, Topics in Cryptology - CT-RSA 2011, volume 6558 of LNCS, pages 237-249. Springer, 2011.

[Sch98] Richard Schroeppel. The Hasty Pudding Cipher. AES submission to NIST, 1998.

$\left[\mathrm{STA}^{+} 14\right]$ Yu Sasaki, Yosuke Todo, Kazumaro Aoki, Yusuke Naito, Takeshi Sugawara, Yumiko Murakami, Mitsuru Matsui, and Shoichi Hirose. Minalpher v1. Submission to the CAESAR competition, 2014.

[Ste12] John Steinberger. Improved Security Bounds for Key-Alternating Ciphers via Hellinger Distance. IACR Cryptology ePrint Archive, Report 2012/481, 2012. Available at http://eprint.iacr.org/ $2012 / 481$.

\section{A Proof of Lemma 2}

The proof of this theorem uses Lemmas 8 and 10 below. In the following, we denote, for every $\alpha, \beta \in\{0,1\}^{n}, \alpha \neq 0, \beta \neq 0$,

$$
\begin{gathered}
\Phi_{\alpha, \beta}(\widetilde{\mathcal{Q}}) \stackrel{\text { def }}{=} N^{2}\left|\sum_{t \in \mathcal{T}} \widehat{1_{\mathcal{Q}_{t}}}(\alpha, \beta)\right|=\left|\sum_{(t, x, y) \in \widetilde{\mathcal{Q}}}(-1)^{\alpha \cdot x \oplus \beta \cdot y}\right| \\
\Phi(\widetilde{\mathcal{Q}}) \stackrel{\text { def }}{=} \max _{\alpha \neq 0, \beta \neq 0} \Phi_{\alpha, \beta}(\widetilde{\mathcal{Q}})
\end{gathered}
$$

where $\mathcal{Q}_{t}$ denotes the set of queries from $\mathcal{A}$ to the permutation $\widetilde{P}(t, \cdot)$ for each $t \in \mathcal{T}$. We also recall four classical results on Fourier analysis over $(\mathbb{Z} / 2 \mathbb{Z})^{n}$. For any set $S \subset\{0,1\}^{n}$ and $\alpha \in\{0,1\}^{n}$,

$$
\begin{aligned}
\widehat{1_{S}}(0) & =\frac{|S|}{N} \\
\sum_{x \in\{0,1\}^{n}} f(x) g(x) & =N \sum_{\alpha \in\{0,1\}^{n}} \widehat{f}(\alpha) \widehat{g}(\alpha) \\
(\widehat{f * g})(\alpha) & =N \widehat{f}(\alpha) \widehat{g}(\alpha) \\
\sum_{\alpha \in\{0,1\}^{n}}\left|\widehat{1_{S}}(\alpha)\right|^{2} & =\frac{|S|}{N} .
\end{aligned}
$$

Lemma 8. For any subset $U, V$ of $\{0,1\}^{n}$, one has

$$
\mu(\widetilde{\mathcal{Q}}, U, V) \leq \frac{q|U||V|}{N}+\Phi(\widetilde{\mathcal{Q}}) \sqrt{|U||V|}
$$


Proof. In the following, we denote

$$
\begin{aligned}
W & =U \times V \\
K & =\left\{(\Gamma(k), k): k \in\{0,1\}^{n}\right\} .
\end{aligned}
$$

Since, for any $t \in \mathcal{T},((x, y), u, v) \in \mathcal{Q}_{t} \times U \times V$ satisfies $x \oplus u=\Gamma(y \oplus v)$ if and only if there exists $k \in\{0,1\}^{n}$ such that

$$
(x, y) \oplus(u, v)=(\Gamma(k), k),
$$

we have

$$
\begin{aligned}
& \mu(\widetilde{\mathcal{Q}}, U, V)=\sum_{t \in \mathcal{T}} \sum_{(x, y) \in\left(\{0,1\}^{n}\right)^{2}} \sum_{(u, v) \in\left(\{0,1\}^{n}\right)^{2}} 1_{\mathcal{Q}_{t}}(x, y) 1_{W}(u, v) 1_{K}(x \oplus u, y \oplus v) \\
& =\sum_{t \in \mathcal{T}} \sum_{(x, y) \in\left(\{0,1\}^{n}\right)^{2}} 1_{\mathcal{Q}_{t}}(x, y) \sum_{(u, v) \in\left(\{0,1\}^{n}\right)^{2}} 1_{W}(u, v) 1_{K}(x \oplus u, y \oplus v) \\
& =\sum_{t \in \mathcal{T}} \sum_{(x, y) \in\left(\{0,1\}^{n}\right)^{2}} 1_{\mathcal{Q}_{t}}(x, y)\left(1_{W} * 1_{K}\right)(x, y) \\
& =N^{2} \sum_{t \in \mathcal{T}} \sum_{(\alpha, \beta) \in\left(\{0,1\}^{n}\right)^{2}} \widehat{\widehat{\mathcal{Q}}_{t}}(x, y)\left(\widehat{1}_{W * 1}\right)(\alpha, \beta) \quad \text { by }(7) \\
& =N^{4} \sum_{t \in \mathcal{T}} \sum_{(\alpha, \beta) \in\left(\{0,1\}^{n}\right)^{2}} \widehat{1_{\mathcal{Q}_{t}}}(x, y) \widehat{1_{W}}(\alpha, \beta) \widehat{1_{K}}(\alpha, \beta) \quad \text { by }(8) \text {. }
\end{aligned}
$$

Separating the principal Fourier coefficient from non-principal ones in the last equality above, we get

$$
\begin{aligned}
& \mu(\widetilde{\mathcal{Q}}, U, V)=N^{4} \sum_{t \in \mathcal{T}} \frac{\left|\mathcal{Q}_{t}\right|}{N^{2}} \frac{|W|}{N^{2}} \frac{|K|}{N^{2}}+N^{4} \sum_{\substack { t \in \mathcal{T} \\
\begin{subarray}{c}{(\alpha, \beta) \\
\neq(0,0){ t \in \mathcal { T } \\
\begin{subarray} { c } { ( \alpha , \beta ) \\
\neq ( 0 , 0 ) } }\end{subarray}} \widehat{\mathcal{Q}_{t}}(x, y) \widehat{1_{W}}(\alpha, \beta) \widehat{1_{K}}(\alpha, \beta) \\
& =\frac{q|U||V|}{N}+N^{4} \sum_{t \in \mathcal{T}} \sum_{(\alpha, \beta) \neq(0,0)} \widehat{1_{\mathcal{Q}_{t}}}(x, y) \widehat{1_{W}}(\alpha, \beta) \widehat{1_{K}}(\alpha, \beta) .
\end{aligned}
$$

Moreover, we have

$$
\begin{aligned}
\widehat{1_{W}}(\alpha, \beta) & =\frac{1}{N^{2}} \sum_{(u, v) \in\left(\{0,1\}^{n}\right)^{2}} 1_{W}(u, v)(-1)^{\alpha \cdot u \oplus \beta \cdot v} \\
& =\frac{1}{N^{2}} \sum_{(u, v) \in\left(\{0,1\}^{n}\right)^{2}} 1_{U}(u) 1_{V}(v)(-1)^{\alpha \cdot u \oplus \beta \cdot v} \\
& =\frac{1}{N^{2}}\left(\sum_{u \in\{0,1\}^{n}} 1_{U}(u)(-1)^{\alpha \cdot u}\right)\left(\sum_{v \in\{0,1\}^{n}} 1_{V}(v)(-1)^{\beta \cdot v}\right) \\
& =\widehat{1_{U}}(\alpha) \widehat{1_{V}}(\beta),
\end{aligned}
$$


and

$$
\begin{aligned}
\widehat{1_{K}}(\alpha, \beta)= & \frac{1}{N^{2}} \sum_{(x, y) \in\left(\{0,1\}^{n}\right)^{2}} 1_{K}(x, y)(-1)^{\alpha \cdot x \oplus \beta \cdot y} \\
= & \frac{1}{N^{2}} \sum_{y \in\{0,1\}^{n}}(-1)^{\alpha \cdot \Gamma(y) \oplus \beta \cdot y} \\
= & \frac{1}{N^{2}} \sum_{y \in\{0,1\}^{n}}(-1)^{\Gamma^{*}(\alpha) \cdot y \oplus \beta \cdot y} \\
= & 0 \text { if } \beta \neq \Gamma^{*}(\alpha) \\
& \frac{1}{N} \text { if } \beta=\Gamma^{*}(\alpha) .
\end{aligned}
$$

Then, injecting the two observations above in (10), we get

$$
\begin{aligned}
\mu(\widetilde{\mathcal{Q}}, U, V) & =\frac{q|U||V|}{N}+N^{3} \sum_{\alpha \neq 0} \widehat{1_{U}}(\alpha) \widehat{1_{V}}\left(\Gamma^{*}(\alpha)\right) \sum_{t \in \mathcal{T}} \widehat{1_{\mathcal{Q}_{t}}}\left(\alpha, \Gamma^{*}(\alpha)\right) \\
& \leq \frac{q|U||V|}{N}+N^{3} \sum_{\alpha \neq 0}\left|\widehat{1_{U}}(\alpha)\right| \cdot\left|\widehat{1_{V}}\left(\Gamma^{*}(\alpha)\right)\right| \cdot\left|\sum_{t \in \mathcal{T}} \widehat{1_{\mathcal{Q}_{t}}}\left(\alpha, \Gamma^{*}(\alpha)\right)\right| \\
& \leq \frac{q|U||V|}{N}+N \Phi(\widetilde{\mathcal{Q}}) \sum_{\alpha \neq 0}\left|\widehat{1_{U}}(\alpha)\right| \cdot\left|\widehat{1_{V}}\left(\Gamma^{*}(\alpha)\right)\right|
\end{aligned}
$$

by definition of $\Phi(\widetilde{\mathcal{Q}})$. By Cauchy-Schwartz,

$$
\begin{aligned}
\sum_{\alpha \neq 0}\left|\widehat{1_{U}}(\alpha)\right| \cdot\left|\widehat{1_{V}}\left(\Gamma^{*}(\alpha)\right)\right| & \leq \sqrt{\sum_{\alpha \in\{0,1\}^{n}}\left|\widehat{1_{U}}(\alpha)\right|^{2}} \sqrt{\sum_{\alpha \in\{0,1\}^{n}}\left|\widehat{1_{V}}\left(\Gamma^{*}(\alpha)\right)\right|^{2}} \\
& =\frac{1}{N} \sqrt{|U||V|},
\end{aligned}
$$

where the last equality follows from (9), so that we get

$$
\mu(\widetilde{\mathcal{Q}}, U, V) \leq \frac{q|U||V|}{N}+\Phi(\widetilde{\mathcal{Q}}) \sqrt{|U||V|}
$$

The following lemma from $\left[\mathrm{CLL}^{+} 14\right]$ will also be needed.

Lemma 9. Let $0 \leq \epsilon<1 / 2$, and let $\mathbf{A}=\left(A_{i}\right)_{1 \leq i \leq q}$ be a sequence of random variables taking values in $\{1,-1\}$. Assume that, for any $1 \leq i \leq q$ and any sequence $\left(a_{1}, \ldots, a_{i-1}\right) \in\{1,-1\}^{i-1}$, one has

$$
\operatorname{Pr}\left[A_{i}=1 \mid\left(A_{1}, \ldots, A_{i-1}\right)=\left(a_{1}, \ldots, a_{i-1}\right)\right] \leq \frac{1}{2}+\epsilon .
$$

Then, for any $\delta \in[0,1]$, one has

$$
\operatorname{Pr}\left[\sum_{i=1}^{q} A_{i} \geq q(2 \epsilon+\delta)\right] \leq e^{-\frac{q \delta^{2}}{12}} .
$$

Now we can prove an upper-bound for $\Phi(\widetilde{\mathcal{Q}})$. 
Lemma 10. Assume that $9 n \leq q \leq N / 2$. Fix an adversary $\mathcal{A}$ making $q$ queries to a random tweakable permutation $\widetilde{P}$ with tweak space $\mathcal{T}$. Let $\widetilde{\mathcal{Q}}$ denote the transcript of the interaction of $\mathcal{A}$ with $\widetilde{P}$. Then

$$
\underset{\widetilde{P}, \omega}{\operatorname{Pr}}\left[\Phi(\widetilde{\mathcal{Q}}) \geq \frac{2 q^{2}}{N}+3 \sqrt{n q}\right] \leq \frac{2}{N},
$$

where the probability is taken over the random choice of $\widetilde{P}$ and the random coins $\omega$ of $\mathcal{A}$.

Proof. In all this proof, $\operatorname{Pr}[\cdot]$ denotes $\operatorname{Pr}_{\widetilde{P}, \omega}[\cdot]$. Fix $\alpha, \beta \in\{0,1\}^{n}, \alpha \neq 0$ and $\beta \neq 0$. Letting $\widetilde{\mathcal{Q}}=\left(\left(t_{1}, x_{1}, y_{1}\right), \ldots,\left(t_{q}, x_{q}, y_{q}\right)\right)$ following the natural ordering of the queries of $\mathcal{A}$, we define the sequence of random variables $\left(A_{i}\right)_{1 \leq i \leq q}$ where $A_{i}=(-1)^{\alpha \cdot x_{i} \oplus \beta \cdot y_{i}}$. Then $\Phi_{\alpha, \beta}(\widetilde{\mathcal{Q}})=\left|\sum_{i=1}^{q} A_{i}\right|$. In order to apply Lemma 9 , we will show that for $1 \leq i \leq q$, and any sequence $\left(a_{1}, \ldots, a_{i-1}\right) \in$ $\{1,-1\}^{i-1}$, we have

$$
p_{i} \stackrel{\text { def }}{=} \operatorname{Pr}\left[A_{i}=1 \mid\left(A_{1}, \ldots, A_{i-1}\right)=\left(a_{1}, \ldots, a_{i-1}\right)\right] \leq \frac{1}{2}+\frac{q}{N} .
$$

The $i$-th query is asked to the permutation $P\left(t_{i}, \cdot\right)$. Assume that that this is a forward query $x_{i}$. Note that the answer $y_{i}$ is distributed uniformly at random on a set of size at least $N-i+1$. Also notice that, once $x_{i}$ is fixed, there are exactly $N / 2 y_{i}^{\prime}$ 's such that $A_{i}=(-1)^{\alpha \cdot x_{i} \oplus \beta \cdot y_{i}}=1$ since $\beta \neq 0$. Similarly, if the $i$-th query is a backward query $y_{i}$, then the answer $x_{i}$ is distributed uniformly at random on a set of size at least $N-i+1$, and once $y_{i}$ is fixed, there are exactly $N / 2 x_{i}$ 's such that $A_{i}=(-1)^{\alpha \cdot x_{i} \oplus \beta \cdot y_{i}}=1$ since $\alpha \neq 0$. Hence we have that

$$
p_{i} \leq \frac{N / 2}{N-i+1} \leq \frac{N}{2(N-q)} \leq \frac{1}{2}+\frac{q}{2(N-q)} \leq \frac{1}{2}+\frac{q}{N}
$$

We can now apply Lemma 9 with $\epsilon=\frac{q}{N}$ and we get, for any $\delta \in[0,1]$,

$$
\operatorname{Pr}\left[\sum_{i=1}^{q} A_{i} \geq \frac{2 q^{2}}{N}+q \delta\right] \leq e^{-\frac{q \delta^{2}}{12}} .
$$

Defining $A_{i}^{\prime}=-A_{i}$, and applying exactly the same reasoning, we get

$$
\operatorname{Pr}\left[\sum_{i=1}^{q} A_{i} \leq-\frac{2 q^{2}}{N}+q \delta\right] \leq e^{-\frac{q \delta^{2}}{12}} .
$$

Thus by a union bound we get

$$
\operatorname{Pr}\left[\Phi_{\alpha, \beta}(\widetilde{\mathcal{Q}}) \geq \frac{2 q^{2}}{N}+q \delta\right] \leq 2 e^{-\frac{q \delta^{2}}{12}}
$$

Note that this holds for any $\alpha \neq 0$ and $\beta \neq 0$. Hence, if we choose $\delta=\sqrt{(12 \ln N) / q}$ (which, assuming $q \geq 9 n$, implies $\delta<1$ ), using $\sqrt{12 \ln 2} \geq 3$, we finally get the result. 


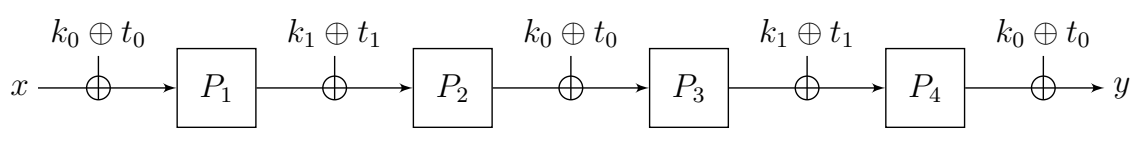

Fig. 4. The 4-round tweakable Even-Mansour construction with a $2 n$-bit key $\left(k_{0}, k_{1}\right)$ and a $2 n$-bit tweak $\left(t_{0}, t_{1}\right)$.

\section{B The Case of $2 n$-bit Tweaks}

In this section, we ask whether the construction of Section 3 can be extended to handle $2 n$-bit tweaks $\left(t_{0}, t_{1}\right)$. We show that the naive way of proceeding, namely alternatively adding $t_{0}$ and $t_{1}$, is insecure for four rounds. In more details, consider the 4-round construction TEM $[n, 4, \mathbf{f}]$ obtained from functions $\mathbf{f}=\left(f_{0}, \ldots, f_{4}\right)$ where $f_{i}\left(\left(k_{0}, k_{1}\right),\left(t_{0}, t_{1}\right)\right)=k_{i \bmod 2} \oplus t_{i \bmod 2}$ and depicted on Figure 4. Fix any tuple of permutations $\mathbf{P}=\left(P_{1}, \ldots, P_{4}\right)$ and let $\widetilde{E}$ be the resulting tweakable block cipher $\widetilde{E}=\mathrm{TEM}^{\mathbf{P}}$. If the adversary sets $t_{1}=0$, then the construction takes the form

$$
\widetilde{E}\left(\left(k_{0}, k_{1}\right),\left(t_{0}, 0\right), x\right)=k_{0} \oplus t_{0} \oplus P_{2}^{\prime}\left(k_{0} \oplus t_{0} \oplus P_{1}^{\prime}\left(k_{0} \oplus t_{0} \oplus x\right)\right),
$$

where

$$
\begin{aligned}
& P_{1}^{\prime}(u)=P_{2}\left(k_{1} \oplus P_{1}(u)\right) \\
& P_{2}^{\prime}(u)=P_{4}\left(k_{1} \oplus P_{3}(u)\right) .
\end{aligned}
$$

In other words, one obtains an equivalent 2-round construction, based on permutations $\left(P_{1}^{\prime}, P_{2}^{\prime}\right)$ that depend only on $k_{1}$, with an $n$-bit key $k_{0}$ and an $n$-bit tweak $t_{0}$, using the simple mixing functions $f_{i}^{\prime}\left(k_{0}, t_{0}\right)=k_{0} \oplus t_{0}$ for $i=0,1,2$. Yet it has been shown in [CS15] that such a construction can be distinguished from random with only four construction queries (and no queries to internal permutation, which is required in our case since $P_{1}^{\prime}$ and $P_{2}^{\prime}$ depend on $k_{1}$ ).

Hence, it seems that at least five rounds are required to obtain a secure construction. 